\title{
Supersymmetry at Colliders
}

\section{Jan Kalinowski}

Faculty of Physics, University of Warsaw, Hoża 69, PL-00681 Warsaw, Poland

E-mail: Jan.Kalinowski@fuw.edu.pl

These lecture notes give an introduction to supersymmetry and its phenomenology at colliders. In the first lecture, after motivating, we discuss supersymmetry formalism and the construction of supersymmetric Lagrangian with scalars, fermions and gauge bosons. The second lecture covers the structure of the Minimal Supersymmetric Standard Model (MSSM). Current experimental limits and the prospects of measurements of MSSM parameters at the LHC are briefly reviewed. Assuming that the LHC finds signals of SUSY, in the last lecture we discuss the exploration of supersymmetry at future Linear Colliders with the ultimate goal of reconstructing the fundamental theory.

Third Linear Collider Physics School 2009 - LCPS2009

August 17 - 232009

Ambleside, UK 


\section{Introduction}

\subsection{Preamble}

These lecture notes are not meant to give a self-contained course of supersymmetry (SUSY) and related phenomenology. It is impossible to cover all these areas in 3 hours of lectures. They are meant to guide the students through the first steps of learning supersymmetry and help them understanding basic phenomenology. The discussion will also be limited to global $\mathrm{N}=1$ SUSY. There are many excellent reviews and text-books that go beyond these limits [1], and the hope is that these notes will encourage students to broaden the knowledge on their own.

After motivating supersymmetry we discuss its formalism in section 2 with the aim of constructing the supersymmetric extension of the Standard Model. The structure of the minimal extension (MSSM) is the subject of the following section. The rest is devoted to phenomenological studies of SUSY at present and future colliders. Since the parameter space of the MSSM is enormous which contains different scenarios for particle physics and cosmology, we have to limit ourselves to the simplest and well-studied that have been performed in the past. Nevertheless, we will try to indicate possible branches and give references to follow some alternative routes. After showing some results of SUSY searches at existing colliders: Tevatron and HERA, the discovery potentials of the main two LHC detectors: ATLAS and CMS, are briefly reviewed. Assuming that SUSY is discovered at LHC we will discuss how experimentation at the ILC will help in revealing the details of the underlying model. Finally we will address the question of reconstructing the SUSY parameters and the mechanism of SUSY breaking.

\subsection{Motivation for supersymmetry}

The Standard Model (SM) provides a precise comprehensive description of the constituents of matter and their interactions [2]. It is a renormalisable quantum field theory based on the local gauge invariance under the $S U(3)_{\text {color }} \times S U(2)_{\text {isospin }} \times U(1)_{\text {hypercharge }} \equiv S U(3)_{c} \times S U(2)_{L} \times$ $U(1)_{Y}$ symmetry transformations. Once $\sim 20$ input parameters are fixed (coupling constants, masses and mixing angles of fermions as well as the $Z$ and Higgs boson masses) explicit and precise calculations can be performed that agree with all experimental observables up to currently probed scale of $\sim 100 \mathrm{GeV}$. In a sense it is the best fundamental theory we ever had. For more than thirty years, since the SM was established in the late 1970's, the experimentalists desperately try to disprove it. Why then we want to go beyond it?

In spite of all its successes the SM cannot be the ultimate theory. The local isospin and hypercharge gauge symmetries have to be spontaneously broken to provide masses to fermions and electroweak gauge bosons. In the SM the sole source of symmetry breaking is an isospin doublet of elementary scalar Higgs fields with the mass parameter squared in the Higgs potential,

$$
V(\phi)=-\mu^{2}|\phi|^{2}+\lambda|\phi|^{4}
$$

arranged to be negative. Since $\mu^{2}$ is a renormalisable parameter of the theory, its value including the sign cannot be computed form the first principles, and assuming $\mu^{2}>0$ does the job. In fact $\mu$ the only dimensionfull parameter of the theory that fixes the mass scale and to fit the data it must be of order electroweak scale $|\mu| \sim 100 \mathrm{GeV}$. The problem is that $\mu^{2}$ receives huge quantum 
corrections via loop diagrams from every particle that couples to the Higgs field. For example, the Higgs couples to the SM fermions with a term in the Lagrangian $-\lambda_{f} \phi \bar{\psi}_{f} \psi_{f}$ and the first order diagram with a fermion loop yields a correction

$$
\delta \mu^{2} \sim-\lambda_{f}^{2} \int d^{4} k\left(\frac{1}{k^{2}-m_{f}^{2}}+\frac{2 m_{f}^{2}}{\left(k^{2}-m_{f}^{2}\right)^{2}}\right)
$$

The first integral is quadratically divergent in the ultraviolet, $-\lambda_{f}^{2} \Lambda^{2}$, where $\Lambda$ as an UV cutoff up to which the SM is considered a valid effective theory. What is $\Lambda$ ? If it is of the order the Planck scale $M_{P l} \sim 10^{19} \mathrm{GeV}$, the correction is some 30 orders of magnitude larger than the required scale. Even if we pretend the SM valid at any scale and use the dimensional regularisation, in which quadratic divergences can be avoided, the next term in eq. (1.2) proportional to $m_{f}^{2}$, which is logarithmic divergent, requires fine tuning to be eliminated as well. Here we should contrast the quantum correction to the scalar field in the above equation to the loop-correction to the fermion propagator, where the logarithmic divergence is proportional the the fermion mass itself. Thus the fermion receives a correction controlled by its mass, and even for $\Lambda=M_{P l}$, the logarithmic correction to the fermion mass is modest. The correction to the scalar is controlled by a mass of a particle circulating in the loop, so is sensitive to the masses of heaviest particles to which the scalar couples, directly on indirectly. Hence the effects of possible heavy states (with masses well above currently explored region) on the Standard Model do not decouple and prevent understanding why $\mu^{2}$ is so low. This is the famous gauge hierarchy problem of the Standard Model.

The origin of the smallness of quantum corrections to fermion masses is linked to the chiral symmetry of the model. For a massless fermion the Lagrangian is invariant under chiral rotations, $\psi \rightarrow e^{-i \gamma_{5} \alpha} \psi$. In the limit of vanishing bare fermion mass, the quantum correction must vanish, hence $\delta m_{\psi} \sim m_{\psi}$, while a scalar field is not protected by any extra symmetry from receiving large corrections.

There have been proposed many paths to solve the hierarchy problem. One is to admit a new dynamics ('technicolor') at a scale close to $1 \mathrm{TeV}$, in which the Higgs is a composite state of strongly interacting techniquarks. Today this approach is disfavored since it is at odds with electroweak measurements. The other path is to invoke a symmetry that would control the size of quantum corrections. The 'little Higgs' models rely on global symmetries which protect the Higgs boson mass against quadratically divergent radiative corrections at one-loop. Models with extra space dimensions postulate a symmetry that connects a scalar field to a gauge field, whose mass is forbidden by gauge symmetry. Supersymmetry postulates a symmetry that connects the scalar field to a fermion field, whose mass is controlled by the chiral symmetry. In fact, supersymmetry is the only mathematically self-consistent UV completion of the Standard Model up to a GUT or Planck scale.

Apart from the hierarchy problem, there are other key questions that the SM does not address:

- why neutrinos are so different from charged fermions?

- what is the origin of matter-antimatter asymmetry?,

- do all forces, including gravity, unify?

- what is the nature of dark matter, dark energy?

All these questions seem to point to new phenomena at a $\mathrm{TeV}$ scale which can experimentally be 
explored soon at the Large Hadron Collider (LHC) and in (hopefully) not too far a future at the International Linear Collider (ILC).

Although each of the above questions could have different origin, it is interesting to note that supersymmetry (SUSY) can provide a common base for all of them. Being almost as old as the SM itself, SUSY [3, 4] still lacks any direct experimental evidence. Why then we do not give up? We should remember, however, that it took some 40 years (since Fermi's time) to formulate the $\mathrm{SM}, 20$ years from the $b$-quark to $t$-quark discovery. It was also clear from the beginning that the required scale to study electroweak theory is $\mathrm{TeV}$, and only after 70 years we are finally getting there. But most important, SUSY turned to be able to beautifully accommodate or explain (at least in the technical sense) some of the outstanding problems of the Standard Model, although it was not invented or designed to do so. For example, SUSY solves the hierarchy problem, explains the gauge coupling unification, provides the radiative electroweak symmetry breaking. It predicts the heavy top quark, provides a perfect candidate for dark matter (DM), offers new ideas on matter-antimatter asymmetry of the universe etc. Moreover, the unique mathematical nature of supersymmetric theories provides us a telescope to physics at the GUT/Planck scale where particle physics meets gravity.

\section{Supersymmetry formalism ${ }^{1}$}

\subsection{Symmetries}

A symmetry is a group of transformations that leaves the action invariant. Symmetries are very interesting at least for two reasons: First, it seems that nature respects many symmetries. Examples are symmetries with respect to space translations or rotations. Second, the Noether theorem states that with each continuous symmetry we can associate a conserved quantity. Symmetry under space translation and rotation imply conservation of momentum and angular momentum.

In a quantum mechanical system, symmetries are defined by a transformation of a state vector $\psi(\vec{x})$. For example, under space translations by a vector $\vec{a}$ and rotations described by three angles $\vec{\vartheta}$ the state $\psi(\vec{x})$ transforms as

$$
\psi(\vec{x}) \rightarrow \psi^{\prime}(\vec{x})=e^{-i \vec{a} \cdot \vec{P}} e^{-i \vec{\vartheta} \cdot \vec{J}} \psi(\vec{x}) .
$$

$J^{i}$ and $P^{i}(i \in\{1,2,3\})$ are called the generators of the rotations and translations, respectively. Although their explicit form depends on the precise nature (spin) of the state, they form an algebra with a definite set of commutation relations

$$
\begin{aligned}
{\left[P^{i}, P^{j}\right] } & =0, \\
{\left[J^{i}, J^{j}\right] } & =i \epsilon^{i j k} J^{k}, \\
{\left[P^{i}, J^{j}\right] } & =i \epsilon^{i j k} P^{k} .
\end{aligned}
$$

In the relativistic quantum theory the symmetry group is enlarged to the Poincaré group consisting of Lorentz transformations and space-time translations

$$
x^{\mu} \rightarrow x^{\prime \mu}=x^{\mu}+\omega^{\mu \nu} x_{\nu}+a^{\mu} .
$$

\footnotetext{
${ }^{1}$ In preparing the first lecture on SUSY formalism I benefited very much from a recent paper by Adrian Signer [5].
} 
The transformation of a field $\Phi(x)$ under the Lorentz transformation and translation of eq.(2.3) can be written as

$$
\Phi(x) \rightarrow \Phi^{\prime}(x)=e^{i a^{\mu} P_{\mu}+i \omega^{\mu \nu} M_{\mu \nu}} \Phi(x) .
$$

This involves six generators of Lorentz transformations $M^{\mu \nu}=-M^{\nu \mu}$, three for rotations $M^{i j}=$ $\epsilon^{i j k} J^{k}$ and three for boosts $M^{0 i}$, and four generators of translations, $P^{\mu}$, three for space $P^{i}$ and one for time $P^{0}$ translations (where Latin indices run $1,2,3$ Greek $\mu, \nu$ run $0,1,2,3$, and $\epsilon^{i j k}$ antisymmetric tensor). Note that the number of coordinates has also been enlarged from $\vec{x}$ to $x^{\mu}$. Again the explicit form of the generators depends on the nature of the field $\Phi$ that act on. The algebra (2.2) in this case is enlarged to the Poincaré algebra

$$
\begin{aligned}
& {\left[P^{\rho}, P^{\sigma}\right]=0,} \\
& {\left[P^{\rho}, M^{\nu \sigma}\right]=i\left(g^{\rho \nu} P^{\sigma}-g^{\rho \sigma} P^{\nu}\right),} \\
& {\left[M^{\mu \nu}, M^{\rho \sigma}\right]=-i\left(g^{\mu \rho} M^{\nu \sigma}+g^{\nu \sigma} M^{\mu \rho}-g^{\mu \sigma} M^{\nu \rho}-g^{\nu \rho} M^{\mu \sigma}\right) .}
\end{aligned}
$$

In quantum theory the role of symmetries is even more important: the presence of a conserved quantity implies the invariance of the action. Apart from space-time transformations there are also so called internal symmetry transformations. These are not transformations in the configuration space, but in the internal space of quantum fields specified by some parameters $\alpha^{a}, \psi \rightarrow \psi^{\prime}=$ $e^{i T^{a} \alpha^{a}} \psi$. The generators $T^{a}$ satisfy a set of commutation relations

$$
\left[T^{a}, T^{b}\right]=i f^{a b c} T^{c}
$$

where $f^{a b c}$ are the structure constants of the underlying symmetry group. For example, the conservation of the electric charge implies the symmetry of the quantum electrodynamics under the change of the phase of the electron field (global gauge transformation). Other examples are baryon number conservation, flavor symmetries or gauge symmetries. Identifying the conserved quantum numbers and corresponding symmetries is a crucial help in constructing theories of fundamental interactions.

The above internal symmetries extend the Poincaré symmetry in a trivial way since the generators of internal symmetries commute with the Poincaré generators. It has been proven by Coleman and Mandula [6] that any symmetry compatible with an interacting relativistic QFT is of the form of a direct product of the Poincaré algebra with an internal symmetry, such as gauge symmetry. However, an implicit assumption in the proof was the bosonic character of generators and their algebra defined by commutation relations. However, when fermionic generators that change the spin of the state by 1/2 unit are allowed the Coleman-Mandula theorem can be avoided and a non-trivial extension of the Poincaré algebra, named supersymmetry, achieved [3]. With the field-theoretic realisation of supersymmetry algebra by Wess and Zumino [4], the detailed exploration of this symmetry and its application to particle physics has begun.

\subsection{Supersymmetry}

In quantum theory a symmetry that links a boson with a fermion [7] is generated by a conserved charge $Q_{\alpha}$ that carries spin-1/2

$$
\left.\left.\left.\left.Q_{\alpha} \mid \text { boson }\right\rangle=\mid \text { fermion }\right\rangle_{\alpha}, \quad Q_{\alpha} \mid \text { fermion }\right\rangle^{\alpha}=\mid \text { boson }\right\rangle,
$$


where $Q_{\alpha}$ is a Weyl spinor; a four-component Dirac spinor is build from $Q_{\alpha}$ and its Hermitian conjugate $\bar{Q}_{\dot{\alpha}}=\left(Q_{\alpha}\right)^{\dagger}$. Here we use a commonly adopted notation that $Q$ is written with undotted, and $\bar{Q}$ with dotted indices $\alpha, \dot{\alpha} \in\{1,2\}$. The conserved charge $Q_{\alpha}$ implies the existence of a conserved 4-vector charge defined by

$$
\left\{Q_{\alpha}, \bar{Q}_{\dot{\beta}}\right\}=2\left(\sigma^{\rho}\right)_{\alpha \dot{\beta}} S_{\rho}
$$

Coleman and Mandula proved that in the relativistic quantum field theory if there exisits an additional conserved 4-vector apart from the 4-momentum, the theory is trivial. Therefore to have a non-trivial supersymmetry we have to set $S_{\rho}=P_{\rho}$, because the RHS of eq.(2.8) can only vanish when both $Q_{\alpha}$ and $\bar{Q}_{\dot{\alpha}}$ annihilate every state in the theory, as can be easily checked by calculating the expectation value of (2.8) in any state for $\alpha=\dot{\beta}$.

If we allow for one set of such fermionic generators, as shown by Haag, Łopuszański and Sohnius [8], the supersymmetric extension of the Poincaré algebra takes the form

$$
\begin{aligned}
{\left[Q_{\alpha}, P^{\rho}\right] } & =0 \\
\left\{Q_{\alpha}, \bar{Q}_{\dot{\beta}}\right\} & =2\left(\sigma^{\rho}\right)_{\alpha \dot{\beta}} P_{\rho}, \\
{\left[M^{\rho \sigma}, Q_{\alpha}\right] } & =-i\left(\sigma^{\rho \sigma}\right)_{\alpha}^{\beta} Q_{\beta}, \\
\left\{Q_{\alpha}, Q_{\beta}\right\} & =\left\{\bar{Q}_{\dot{\alpha}}, \bar{Q}_{\dot{\beta}}\right\}=0,
\end{aligned}
$$

where $\left(\sigma^{\rho}\right)_{\alpha \dot{\beta}},\left(\sigma^{\rho \sigma}\right)_{\alpha}^{\beta}$ are defined in the following subsection. One set of such generators corresponds to $N=1$ supersymmetry. Adding more sets of fermionic operators we would end up in $N>1$ supersymmetry, which however is ruled out as a low-energy $(\sim 1 \mathrm{TeV})$ extension of the Standard Model.

Since $Q_{\alpha}$ is a 'square-root' of the energy-momentum vector, the fermionic operator must act on every field in the theory. Thus supersymmetry forces us to double number of known particles: every particle must have a superpartner of opposite statistics and spin different by $1 / 2$. We will see later that in the supersymmetric extension of the SM we will also have to include a second Higgs doublet (and their supersymmetric partners).

\subsection{Technicalities}

In order to be able to construct supersymmetric theories in an efficient way we need to discuss first some conventions and technicalities. We will follow conventions used in Ref. [5].

In supersymmetric theories it is convenient to work with two-component Weyl spinors $\psi_{\alpha}, \chi_{\alpha}, \ldots$ The Hermitian conjugate spinors will be denoted by the bar over the symbol and the dotted index, i.e. $\bar{\psi}_{\dot{\alpha}} \equiv\left(\psi_{\alpha}\right)^{\dagger}$. If $\psi_{\alpha}$ transforms as $\left\{\frac{1}{2}, 0\right\}$ under Lorentz, then $\bar{\psi}$ transforms as $\left\{0, \frac{1}{2}\right\}$. Using the Pauli matrices we define

$$
\left(\sigma^{\mu}\right)_{\alpha \dot{\alpha}} \equiv\left\{1, \sigma^{1}, \sigma^{2}, \sigma^{3}\right\}_{\alpha \dot{\alpha}} \quad\left(\bar{\sigma}^{\mu}\right)^{\dot{\alpha} \beta} \equiv,\left\{1,-\sigma^{1},-\sigma^{2},-\sigma^{3}\right\}^{\dot{\alpha} \beta} .
$$

It is easy to check the identity

$$
\left(\sigma^{\mu}\right)_{\alpha \dot{\alpha}}\left(\bar{\sigma}^{\nu}\right)^{\dot{\alpha} \alpha}=\operatorname{Tr}\left(\sigma^{\mu} \bar{\sigma}^{\nu}\right)=2 g^{\mu \nu} .
$$


The Dirac $\gamma$ matrices are then defined as

$$
\gamma^{\mu} \equiv\left(\begin{array}{cc}
0 & \sigma^{\mu} \\
\bar{\sigma}^{\mu} & 0
\end{array}\right), \quad \gamma^{5} \equiv i \gamma^{0} \gamma^{1} \gamma^{2} \gamma^{3}=\left(\begin{array}{cc}
-1 & 0 \\
0 & 1
\end{array}\right)
$$

since the usual commutation relations $\left\{\gamma^{\mu}, \gamma^{\nu}\right\}=2 g^{\mu \nu}$ is a consequence of (2.14) (we use $g^{\mu \nu}=$ $\left.g_{\mu \nu}=\operatorname{diag}\{1,-1,-1,-1\}\right)$.

The spinor indices can be raised/lowered as follows

$$
\psi_{\alpha}=\epsilon_{\alpha \beta} \psi^{\beta}, \quad \psi^{\alpha}=\epsilon^{\alpha \beta} \psi_{\beta}, \bar{\psi}_{\dot{\alpha}}=\epsilon_{\dot{\alpha} \dot{\beta}} \bar{\psi}^{\dot{\beta}}, \bar{\psi}^{\dot{\alpha}}=\epsilon^{\dot{\alpha} \dot{\beta}} \bar{\psi}_{\dot{\beta}},
$$

where the totally antisymmetric $\epsilon$ tensor is defined as $\epsilon^{12}=\epsilon_{21}=-\epsilon^{21}=-\epsilon_{12} \equiv 1,0$ otherwise. Other useful relations are

$$
\begin{array}{lc}
\epsilon^{\alpha \gamma} \epsilon_{\gamma \beta}=\delta_{\beta}^{\alpha}, \quad \epsilon_{\alpha \gamma} \epsilon^{\gamma \beta}=\delta_{\alpha}^{\beta}, & \epsilon^{\alpha \gamma} \epsilon_{\beta \delta}=\delta_{\delta}^{\alpha} \delta_{\beta}^{\gamma}-\delta_{\beta}^{\alpha} \delta_{\delta}^{\gamma}, \\
\left(\sigma^{\mu \nu}\right)_{\alpha}^{\beta} \equiv \frac{1}{4}\left(\sigma^{\mu} \bar{\sigma}^{\nu}-\sigma^{\nu} \bar{\sigma}^{\mu}\right)_{\alpha}^{\beta}, & \left(\bar{\sigma}^{\mu \nu}\right)_{\dot{\beta}}^{\dot{\alpha}} \equiv \frac{1}{4}\left(\bar{\sigma}^{\mu} \sigma^{\nu}-\bar{\sigma}^{\nu} \sigma^{\mu}\right)_{\dot{\beta}}^{\dot{\alpha}}, \\
\left(\sigma^{\mu} \bar{\sigma}^{\nu}+\sigma^{\nu} \bar{\sigma}^{\mu}\right)_{\alpha}^{\beta}=2 g^{\mu \nu} \delta_{\alpha}^{\beta}, & \left(\bar{\sigma}^{\mu} \sigma^{\nu}+\bar{\sigma}^{\nu} \sigma^{\mu}\right)_{\dot{\beta}}^{\dot{\alpha}}=2 g^{\mu \nu} \delta_{\dot{\beta}}^{\dot{\alpha}} .
\end{array}
$$

The Dirac four component spinor $\Psi$ can be constructed from two Weyl spinors $\psi$ and $\chi$ as

$$
\Psi=\left(\begin{array}{c}
\psi_{\alpha} \\
\bar{\chi}^{\dot{\alpha}}
\end{array}\right), \quad \bar{\Psi}=\left(\chi^{\alpha}, \bar{\psi}_{\dot{\alpha}}\right),
$$

where the bar over a Dirac spinor denotes the usual Dirac adjoint $\bar{\Psi} \equiv \Psi^{\dagger} \gamma^{0}$. Note the different position of the indices in the above equation.

The chirality projection operators acting on a Dirac spinor

$$
P_{L} \Psi \equiv \frac{1}{2}\left(1-\gamma_{5}\right) \Psi=\psi_{\alpha}, \quad P_{R} \Psi \equiv \frac{1}{2}\left(1+\gamma_{5}\right) \Psi=\bar{\chi}^{\dot{\alpha}}
$$

imply that $\psi_{\alpha}$ and $\bar{\chi}^{\dot{\alpha}}$ are, respectively, left- and right-handed Weyl spinors.

We will use the following summation convention for the spinor indices

$$
\begin{aligned}
& \chi \psi \equiv \chi^{\alpha} \psi_{\alpha}=\chi^{\alpha} \epsilon_{\alpha \beta} \psi^{\beta} \\
& \bar{\chi} \bar{\psi} \equiv \bar{\chi}_{\dot{\alpha}} \bar{\psi}^{\dot{\alpha}}=\bar{\chi}_{\dot{\alpha}} \epsilon^{\dot{\alpha} \dot{\beta}} \bar{\psi}_{\dot{\beta}} .
\end{aligned}
$$

Since the Fermi fields anticommute, we have $\chi^{\alpha} \psi_{\alpha}=\chi^{\alpha} \epsilon_{\alpha \beta} \psi^{\beta}=-\psi^{\beta} \epsilon_{\alpha \beta} \chi^{\alpha}=\psi^{\beta} \epsilon_{\beta \alpha} \chi^{\alpha}=\psi \chi$, and likewise $\bar{\chi} \bar{\psi}=\bar{\psi} \bar{\chi}$, as needed since these products are Lorentz invariants. Again pay attention to the different position of the dotted and undotted indices in the definition of the products.

The Dirac bilinears can now be written in terms of Weyl bilinears. For example, for the Dirac fields

$$
\Phi=\left(\begin{array}{c}
\lambda_{\alpha} \\
\bar{\phi}^{\dot{\alpha}}
\end{array}\right), \quad \bar{\Psi}=\left(\begin{array}{ll}
\chi^{\alpha} & \bar{\psi}_{\dot{\alpha}}
\end{array}\right),
$$

the Lorentz covariant expressions can be written in two-component notation as follows:

$$
\begin{aligned}
\bar{\Psi} \Phi & =\chi \lambda+\bar{\psi} \bar{\phi}=\chi^{\alpha} \lambda_{\alpha}+\bar{\psi}_{\dot{\alpha}} \bar{\phi}^{\dot{\alpha}} \\
\bar{\Psi} \gamma^{\mu} \Phi & =\chi \sigma^{\mu} \bar{\phi}-\lambda \sigma^{\mu} \bar{\psi}=\chi^{\alpha}\left(\sigma^{\mu}\right)_{\alpha \dot{\alpha}} \bar{\phi}^{\dot{\alpha}}-\lambda^{\alpha}\left(\sigma^{\mu}\right)_{\alpha \dot{\alpha}} \bar{\psi}^{\dot{\alpha}} .
\end{aligned}
$$


As a result, the standard Lagrangian for a free Dirac spinor can be written in terms of Weyl spinors as

$$
i \bar{\Psi} \gamma^{\mu} \partial_{\mu} \Psi-m \bar{\Psi} \Psi=i \chi \sigma^{\mu} \partial_{\mu} \bar{\chi}+i \psi \sigma^{\mu} \partial_{\mu} \bar{\psi}-m \chi \psi-m \bar{\psi} \bar{\chi}
$$

where integration by parts $-i\left(\partial_{\mu} \psi\right) \sigma^{\mu} \bar{\psi}=i \psi \sigma^{\mu} \partial_{\mu} \bar{\psi}$ has been used. In the case of a Majorana spinor a single Weyl spinor is sufficient

$$
\Psi_{M}=\left(\begin{array}{c}
\psi_{\alpha} \\
\bar{\psi}^{\dot{\alpha}}
\end{array}\right), \quad \bar{\Psi}_{M}=\left(\psi^{\alpha}, \bar{\psi}_{\dot{\alpha}}\right),
$$

and the standard Lagrangian written in terms of a Weyl spinor reads

$$
\frac{i}{2} \bar{\Psi}_{M} \gamma^{\mu} \partial_{\mu} \Psi_{M}-\frac{m}{2} \bar{\Psi}_{M} \Psi_{M}=\frac{i}{2}\left(\psi \sigma^{\mu} \partial_{\mu} \bar{\psi}-\left(\partial_{\mu} \psi\right) \sigma^{\mu} \bar{\psi}\right)-\frac{m}{2}(\psi \psi+\bar{\psi} \bar{\psi})
$$
$\bar{\theta}^{\dot{\alpha}}$

We also introduce a two-component Grassmann spinor variable $\theta^{\alpha}$ and its Hermitian conjugate

$$
\theta^{\alpha}=\left(\begin{array}{c}
\theta^{1} \\
\theta^{2}
\end{array}\right), \quad \bar{\theta}^{\dot{\alpha}}=\left(\begin{array}{c}
\bar{\theta}^{1} \\
\bar{\theta}^{2}
\end{array}\right),
$$

with each entry being a Grassmann variable (which can be thought of as an anticommuting complex number), i.e. $\left\{\theta^{\alpha}, \theta^{\beta}\right\}=\left\{\theta^{\alpha}, \bar{\theta}^{\dot{\beta}}\right\}=\left\{\bar{\theta}^{\dot{\alpha}}, \bar{\theta}^{\dot{\beta}}\right\}=0(\alpha, \beta \in\{1,2\}$. The conventions for raising/lowering indices and products of Grassmann variables are the same as for spinors. For example, a product of a Grassmann component $\theta_{1}$ vanishes, $\theta_{1} \theta_{1}=0$, but a product of a Grassmann coordinate with itself is $\theta \theta=\theta^{1} \theta_{1}+\theta^{2} \theta_{2}=-2 \theta^{1} \theta^{2}$ and does not vanish. But adding one more factor of $\theta_{\alpha}$ does give zero.

The derivatives with respect to a Grassmann variable are defined as follows:

$$
\partial_{\alpha} \equiv \frac{\partial}{\partial \theta^{\alpha}}, \quad \bar{\partial}_{\dot{\alpha}} \equiv \frac{\partial}{\partial \bar{\theta}^{\dot{\alpha}}} .
$$

Using the rules for raising/lowering indices, $\partial^{\alpha} \theta_{\beta}=-\delta_{\beta}^{\alpha}, \bar{\partial}^{\dot{\alpha}} \equiv \epsilon^{\dot{\alpha} \dot{\beta}} \bar{\partial}_{\dot{\beta}}$, and we find

$$
\begin{aligned}
& \partial_{\alpha} \theta^{\beta}=\delta_{\alpha}^{\beta}, \quad \partial^{\alpha} \theta_{\beta}=-\delta_{\beta}^{\alpha}, \quad \partial^{\alpha} \theta^{\beta}=\epsilon^{\alpha \beta}, \quad \partial_{\alpha} \theta_{\beta}=-\epsilon_{\alpha \beta}, \\
& \bar{\partial}_{\dot{\alpha}} \bar{\theta}^{\dot{\beta}}=\delta_{\dot{\alpha}}^{\dot{\beta}} ;, \quad \bar{\partial}^{\dot{\alpha}} \bar{\theta}_{\dot{\beta}}=-\delta_{\dot{\beta}}^{\dot{\alpha}}, \quad \bar{\partial}^{\dot{\alpha}} \bar{\theta}^{\dot{\beta}}=\epsilon^{\dot{\alpha} \dot{\beta}}, \quad \bar{\partial}_{\dot{\alpha}} \bar{\theta}_{\dot{\beta}}=-\epsilon_{\dot{\alpha} \dot{\beta}},
\end{aligned}
$$

and that the derivatives also anticommute with other Grassmann variables

$$
\partial_{\alpha}(\theta \theta)=\left(\partial_{\alpha} \theta^{\beta}\right) \theta_{\beta}-\theta^{\beta}\left(\partial_{\alpha} \theta_{\beta}\right)=\delta_{\alpha}^{\beta} \theta_{\beta}-\theta^{\beta}\left(-\epsilon_{\alpha \beta}\right)=\theta_{\alpha}+\epsilon_{\alpha \beta} \theta^{\beta}=2 \theta_{\alpha}
$$

and similarly $\partial^{\alpha}(\theta \theta)=2 \theta^{\alpha}, \bar{\partial}_{\dot{\alpha}}(\bar{\theta} \bar{\theta})=-2 \bar{\theta}_{\dot{\alpha}}, \bar{\partial}^{\dot{\alpha}}(\bar{\theta} \bar{\theta})=-2 \bar{\theta}^{\dot{\alpha}}$.

Finally, integration with respect to Grassmann variables is defined as

$$
\int \mathrm{d} \theta^{1} \theta^{1}=-\int \theta^{1} \mathrm{~d} \theta^{1}=1, \quad \int \mathrm{d} \theta^{1}=0,
$$

which in practise is the same as differentiation. For convenience we introduce the following notation

$$
\begin{aligned}
& \mathrm{d}^{2} \theta \equiv-\frac{1}{4} \epsilon_{\alpha \beta} \mathrm{d} \theta^{\alpha} \mathrm{d} \theta^{\beta} \\
& \mathrm{d}^{2} \bar{\theta} \equiv-\frac{1}{4} \epsilon_{\dot{\alpha} \dot{\beta}} \mathrm{d} \bar{\theta}^{\dot{\alpha}} \mathrm{d} \bar{\theta}^{\dot{\beta}}
\end{aligned}
$$




\subsection{Superspace and superfields}

The concept of superspace and superfields, although not necessary, is very useful in building supersymmetric theories. We extend the configuration space to superspace

$$
x=\left(x^{\mu}\right) \rightarrow X=\left(x^{\mu}, \theta^{\alpha}, \bar{\theta}^{\dot{\alpha}}\right) .
$$

The convenience of introducing superspace can be parallelled to the convenience of introducing four-vectors $\vec{x} \rightarrow x=\left(x^{\mu}\right)=(t, \vec{x})$ when extending the group of space translations and rotations to a full Poincare group of symmetry transformations: if all Lorentz indices are contracted, the expression is invariant under Lorentz transformations. Since we added new generators $Q_{\alpha}$ and $\bar{Q}_{\dot{\alpha}}$ we will need a matching set of Grassmann coordinates $\theta^{\alpha}$ and $\bar{\theta}^{\dot{\alpha}}$. We will take the coordinates $\theta_{\alpha}$ to transform like two-component Weyl spinors.

Note that with the help of Grassmann coordinates we can formally write the super Poincare algebra in terms of commutator relations, e.g. eq.(2.10) takes the form

$$
[\theta Q, \bar{\theta} \bar{Q}] \equiv\left[\theta^{\alpha} Q_{\alpha}, \bar{\theta}_{\dot{\alpha}} \bar{Q}^{\dot{\alpha}}\right]=2 \theta \sigma^{\mu} \bar{\theta} P_{\mu}
$$

Since we have extended the configuration space to superspace we will consider fields that also depend on Grassmann coordinates, $\Phi(X)=\Phi\left(x^{\mu}, \theta^{\alpha}, \bar{\theta}^{\dot{\alpha}}\right)$. Such fields are called superfields. Since the product of the same Grassmann variable vanishes, the Taylor expansion in Grassmann coordinates terminates very quickly. For example, for a superfield $\Phi\left(x^{\mu}, \theta^{\alpha}\right)$ we have

$$
\Phi(x, \theta)=\phi(x)+\sqrt{2} \theta^{\alpha} \psi_{\alpha}(x)-\theta^{\alpha} \theta_{\alpha} F(x),
$$

i.e. it parameterized by two complex scalar $\phi(x)$ and $F(x)$, and one complex spinor $\psi_{\alpha}(x)$ functions of space-time coordinates $x$ (a factor $\sqrt{2}$ and a minus sign in eq.(2.38) is a convention). Note that the mass dimension of the Grassmann coordinate is given by $[\theta]=[\bar{\theta}]=-1 / 2$ to account for different dimensions of scalar and spinor functions $\phi$ and $\psi$, whereas for ordinary space-time components we have $[x]=-1$. Thus, assuming the mass dimension of the superfield $[\Phi]=1$, the mass dimension of the component fields in the above equation are $[\phi]=1,[\psi]=3 / 2$ and $[F]=2$. Note that $F$ does not have the usual mass dimension of a scalar field. We will see later that the $F$ component field is unphysical and is called an auxiliary field.

Now we need to find the transformation properties of super coordinates and superfields under susy transformations. Consider a SUSY transformation

$$
S(a, \zeta, \bar{\zeta}) \equiv e^{i\left(\zeta^{\alpha} Q_{\alpha}+\bar{\zeta}_{\dot{\alpha}} \bar{Q}^{\dot{\alpha}}+a^{\mu} P_{\mu}\right)}
$$

parameterized by a four-vector $a^{\mu}$, and a Grassmann variable $\zeta^{\alpha}$ (for simplicity we neglect Lorentz boosts and space rotations). With $\zeta=\bar{\zeta}=0$ the transformation reduces to a translation under which a quantum field transforms as

$$
\phi(x) \rightarrow S(a, 0,0) \phi(x) S^{-1}(a, 0,0)=e^{i a^{\mu} P_{\mu}} \phi(x) e^{-i a^{\mu} P_{\mu}}=\phi(x+a) .
$$

The differential form of the momentum operator $P^{\mu}=i \partial^{\mu}$ is then found by Taylor expanding both sides of

$$
\phi(x+a)=e^{-i a^{\mu} P_{\mu}} \phi(x)
$$


and comparing the coefficients of the infinitesimal $a^{\mu}$. One should be aware of a convention commonly used for denoting with the same letter different objects. In the above expression $P_{\mu}$ is a differential operator acting on a function $\phi(x)$, while in eq.(2.40) $P_{\mu}$ is an operator in the Fock space of field operators $\phi$.

Let us repeat the same steps with non-zero $\zeta, \bar{\zeta}$ to find the differential forms of the SUSY generators $Q$ and $\bar{Q}$. Combining two SUSY transformations we obtain

$$
S(a, \zeta, \bar{\zeta}) S(x, \theta, \bar{\theta})=S\left(x^{\mu}+a^{\mu}+i \zeta \sigma^{\mu} \bar{\theta}-i \theta \sigma^{\mu} \bar{\zeta}, \theta+\zeta, \bar{\theta}+\bar{\zeta}\right)
$$

as can easily be checked by using the Baker-Campbell-Hausdorff formula $e^{A} e^{B}=e^{A+B+[A, B] / 2}$ when $[A,[A, B]]=0$. Thus, starting from a point $X=\left(x^{\mu}, \theta^{\alpha}, \bar{\theta}^{\dot{\alpha}}\right)$ in superspace, under a SUSY transformation of eq.(2.42), we have

$$
X \rightarrow X^{\prime}=\left(x^{\mu}+a^{\mu}+i \zeta \sigma^{\mu} \bar{\theta}-i \theta \sigma^{\mu} \bar{\zeta}, \theta+\zeta, \bar{\theta}+\bar{\zeta}\right)
$$

Note that even for $a^{\mu}=0$, a translation is induced under eq.(2.39).

Let us now consider a superfield $\Phi\left(x^{\mu}, \theta^{\alpha}, \bar{\theta}^{\dot{\alpha}}\right)$ under the SUSY transformation eq.(2.39)

$$
\begin{gathered}
\Phi(x, \theta, \bar{\theta}) \rightarrow e^{i\left(\zeta^{\alpha} Q_{\alpha}+\bar{\zeta}_{\dot{\alpha}} \bar{Q}^{\dot{\alpha}}+a^{\mu} P_{\mu}\right)} \Phi(x, \theta, \bar{\theta}) e^{-i\left(\zeta^{\alpha} Q_{\alpha}+\bar{\zeta}_{\dot{\alpha}} \bar{Q}^{\dot{\alpha}}+a^{\mu} P_{\mu}\right)} \\
=\Phi\left(x^{\mu}+a^{\mu}+i \zeta \sigma^{\mu} \bar{\theta}-i \theta \sigma^{\mu} \bar{\zeta}, \theta+\zeta, \bar{\theta}+\bar{\zeta}\right) .
\end{gathered}
$$

Expanding both sides of

$$
\Phi\left(x^{\mu}+a^{\mu}+i \zeta \sigma^{\mu} \bar{\theta}-i \theta \sigma^{\mu} \bar{\zeta}, \theta+\zeta, \bar{\theta}+\bar{\zeta}\right)=e^{-i\left(\zeta^{\alpha} Q_{\alpha}+\bar{\zeta}_{\dot{\alpha}} \bar{Q}^{\dot{\alpha}}+a^{\mu} P_{\mu}\right)} \Phi(x, \theta, \bar{\theta})
$$

and comparing the coefficients of the infinitesimal parameters $a, \zeta$ and $\bar{\zeta}$ we find

$$
\begin{aligned}
P_{\mu} & =i \partial_{\mu}, \\
Q_{\alpha} & =i \partial_{\alpha}-\sigma_{\alpha \dot{\alpha}}^{\mu} \bar{\theta}^{\dot{\alpha}} \partial_{\mu}, \\
\bar{Q}_{\dot{\alpha}} & =-i \bar{\partial}_{\dot{\alpha}}+\theta^{\alpha} \sigma_{\alpha \dot{\alpha}}^{\mu} \partial_{\mu} .
\end{aligned}
$$

In the construction of gauge theories a very useful tool is the covariant derivative defined such that the field and its covariant derivative transform in the same way under gauge transformation. Therefore we define the covariant derivatives as

$$
D_{\alpha} \equiv \partial_{\alpha}-i \sigma_{\alpha \dot{\alpha}}^{\mu} \bar{\theta}^{\dot{\alpha}} \partial_{\mu} \quad \bar{D}_{\dot{\alpha}} \equiv \bar{\partial}_{\dot{\alpha}}-i \theta^{\alpha} \sigma_{\alpha \dot{\alpha}}^{\mu} \partial_{\mu}
$$

We leave to the reader to check that indeed $\Phi$ and $D_{\alpha} \Phi$ (and $\bar{D}_{\dot{\alpha}} \Phi$ ) transform in the same way under SUSY and that e.g. $\left\{D_{\alpha}, Q_{\beta}\right\}=\left\{D_{\alpha}, \bar{Q}_{\dot{\beta}}\right\}=0$, or refer to [5].

\subsection{Chiral superfields}

We have seen that the superfield $\Phi\left(x^{\mu}, \theta^{\alpha}\right)$ in eq.(2.38) is parameterized in terms of a complex function $\phi$ and a chiral spinor $\psi$ (and an additional complex scalar $F$ that will turn to be an auxiliary field). Therefore it can be used to implement the quark or lepton and their scalar superpartners in a supersymmetric construction. A more general superfield $\Phi(x, \theta, \bar{\theta})$ has too many degrees of freedom for that purpose. To reduce the number of degrees of freedom in a self consistent way 
the covariant derivatives $D_{\alpha}$ and $\bar{D}_{\dot{\alpha}}$ can be used. A superfield $\Phi(x, \theta, \bar{\theta})$ is called a left-chiral superfield $(\mathrm{LcSF})$ if $\bar{D}_{\dot{\alpha}} \Phi(x, \theta, \bar{\theta})=0$. This property is invariant under SUSY transformation. It is also interesting to note that the product of LcSF is also the LcSF. The right-chiral superfields (RcSF) are defined by the condition $D_{\alpha} \Phi(x, \theta, \bar{\theta})=0$. Obviously, if $\Phi(x, \theta, \bar{\theta})$ is a LcSF, then $\Phi^{\dagger}$ is the RcSF.

Since $\bar{D}_{\dot{\alpha}} \theta^{\alpha}=0$ and $\bar{D}_{\dot{\alpha}} y^{\mu}=0$, where $y^{\mu} \equiv x^{\mu}-i \theta \sigma^{\mu} \bar{\theta}$, the most general function that satisfies satisfies $\bar{D}_{\dot{\alpha}} \Phi=0$ has the form

$$
\Phi(y, \theta) \equiv \phi(y)+\sqrt{2} \theta \psi(y)-\theta \theta F(y)
$$

$\sqrt{2}$ and the minus sign are conventions. Expanding this back in $x, \theta$ and $\bar{\theta}$ we obtain

$$
\begin{aligned}
\Phi(x, \theta, \bar{\theta}) & =\phi(x)+\sqrt{2} \theta \psi(x)-i \theta \sigma^{\mu} \bar{\theta} \partial_{\mu} \phi(x)+\frac{i}{\sqrt{2}}(\theta \theta)\left(\partial_{\mu} \psi(x) \sigma^{\mu} \bar{\theta}\right) \\
& -\frac{1}{4}(\theta \theta)(\bar{\theta} \bar{\theta}) \partial^{\mu} \partial_{\mu} \phi(x)-(\theta \theta) F(x)
\end{aligned}
$$

The spinors $\psi$ of a LcSF willrepresent the left-handed quarks and leptons of a SUSY extension of the Standard Model and the $\phi$ fields their supersymmetric partners, the squarks and sleptons. The Higgs bosons and their SUSY partners (higgsinos) will also form chiral superfields.

Using the explicit representation of $Q$ and $\bar{Q}$ on the LcSF, we find the explicit form of SUSY transformations of the component fields $\phi(x) \rightarrow \phi(x)+\delta \phi(x)$ etc..,

$$
\begin{aligned}
\delta \phi & =\sqrt{2} \zeta \psi \\
\delta \psi_{\alpha} & =-\sqrt{2} F \zeta_{\alpha}-i \sqrt{2} \sigma_{\alpha \dot{\alpha}}^{\mu} \bar{\zeta}^{\dot{\alpha}} \partial_{\mu} \phi \\
\delta F & =-i \sqrt{2} \partial_{\mu} \psi \sigma^{\mu} \bar{\zeta}=\partial_{\mu}\left(-i \sqrt{2} \psi \sigma^{\mu} \bar{\zeta}\right) .
\end{aligned}
$$

As expected, the change in the bosonic (fermionic) component fields is proportional to the fermionic (bosonic) fields. The important point is that $\delta F$ is a total derivative. Therefore the $F$ component of the LcSF (or products of LcSF) can be used to construct SUSY Lagrangians. They can easily be singled out by integrating over Grassmann variables, since from the definition eq.(2.33) we find

$$
F(x)=\int \Phi(x, \theta \bar{\theta}) \mathrm{d}^{2} \theta=[\Phi(x, \theta, \bar{\theta})]_{\theta \theta},
$$

where on the RHS we introduce a common notation to denote the $\theta \theta$ component of the argument.

\subsection{Vector superfields}

To construct the supersymmetric gauge theory we need a superfield with a spin 1 vector component $v_{\mu}$, which is a real field. A superfield $V(x, \theta, \bar{\theta})$ defined by the constraint $V(x, \theta, \bar{\theta})=$ $V^{\dagger}(x, \theta, \bar{\theta})$, called a vector superfield (VSF), has a required component. Expanding $V$ in terms of components we find

$$
\begin{aligned}
V(x, \theta, \bar{\theta}) & =c(x)+i \theta \chi(x)-i \bar{\theta} \bar{\chi}(x)+\theta \sigma^{\mu} \bar{\theta} v_{\mu}(x)+i(\theta \theta) N(x)-i(\bar{\theta} \bar{\theta}) N^{\dagger}(x) \\
& +i(\theta \theta) \bar{\theta}\left(\bar{\lambda}(x)+\frac{i}{2} \partial_{\mu} \chi(x) \sigma^{\mu}\right)-i(\bar{\theta} \bar{\theta}) \theta\left(\lambda(x)-\frac{i}{2} \sigma^{\mu} \partial_{\mu} \bar{\chi}(x)\right) \\
& +\frac{1}{2}(\theta \theta)(\bar{\theta} \bar{\theta})\left(D(x)-\frac{1}{2} \partial^{\mu} \partial_{\mu} c(x)\right)
\end{aligned}
$$


Note that the definition $V=V^{\dagger}$ is invariant under SUSY transformations, as required. The factors $i$ and some overall signs in the above expansion are simply conventions. The component fields $c, D$ and $v$ are real. Apart from the vector component field $v_{\mu}$ that we wanted, the vector superfield also contains two fermions, $\chi$ and $\lambda$ and a whole set of scalar fields. If we assume the mass dimension of the vector superfield $[V]=0$, the various component fields have dimensions $[c]=0,[\chi]=1 / 2$, $[v]=[N]=1,[\lambda]=3 / 2$ and $[D]=2$. Only $v$ and $\lambda$ have the expected mass dimensions. As in the case of the LcSF, all other component fields will turn out to be unphysical. In fact, when we generalize the gauge symmetry to the supersymmetric case, the component fields $c, N$ and $\lambda$ can be 'gauged away'.

The transformation properties of the component fields of $V$ under SUSY transformations can be found similarly to the case of a LcSF. Here we will limit ourselves to noting that the $D(x)$ component field transforms $D \rightarrow D+\delta D$ with

$$
\delta D=\zeta \sigma^{\mu} \partial_{\mu} \bar{\lambda}(x)+\partial_{\mu} \lambda(x) \sigma^{\mu} \bar{\zeta}=\partial_{\mu}\left(\zeta \sigma^{\mu} \bar{\lambda}(x)+\lambda(x) \sigma^{\mu} \bar{\zeta}\right)
$$

As for the $F$ component field of a chiral superfield, the change in the $D$ field of a VSF is a total derivative. This will again be exploited in constructing supersymmetric Lagrangians by singling out the $D$ components

$$
D(x)=\int V(x, \theta, \bar{\theta}) \mathrm{d}^{2} \theta \mathrm{d}^{2} \bar{\theta}=[V(x, \theta, \bar{\theta})]_{\theta \theta \bar{\theta} \bar{\theta}} .
$$

\subsection{Gauge symmetry}

In the Standard Model the vector fields are the Yang-Mills gauge fields. Therefore we need to combine the gauge symmetry with SUSY. Under the local gauge transformation the vector fields are shifted by a derivative of a scalar gauge function $f$,

$$
v_{\mu} \rightarrow v_{\mu}+\partial_{\mu} f
$$

Recalling that the chiral supermultiplet contains a term $i \theta \sigma^{\mu} \bar{\theta} \partial_{\mu} \phi(x)$, eq.(2.51), we can try to define a gauge transformation of a vector superfield as

$$
V \rightarrow V^{\prime}=V-i \Lambda^{\dagger}+i \Lambda
$$

where $\Lambda$ is a LcSF. However, since the mass dimension of a VSF is zero, the $\Lambda$ superfield has to have a 'wrong' mass dimension as well, $[\Lambda]=0$. Therefore its scalar and spinor components must have mass dimension 0 and $1 / 2$, respectively, and cannot represent physical fields. In fact they can be chosen to eliminate the unphysical component fields from the vector superfield. If we choose $\Lambda(x, \theta, \bar{\theta})$ as in eq. (2.51) but with the replacements $\psi \rightarrow-\chi / \sqrt{2}, F \rightarrow N$ and $\operatorname{Im}(\varphi) \rightarrow c / 2$, then $V^{\prime}$ reduces to a simple expression

$$
V^{\prime} \equiv V_{\mathrm{WZ}}(x, \theta, \bar{\theta})=\theta \sigma^{\mu} \bar{\theta} v_{\mu}(x)+i(\theta \theta) \bar{\theta} \bar{\lambda}(x)-i(\bar{\theta} \bar{\theta}) \theta \lambda(x)+\frac{1}{2}(\theta \theta)(\bar{\theta} \bar{\theta}) D(x)
$$

Thus, in the so called Wess-Zumino gauge, the vector field exposes only four (three in $v_{\mu}$ one in $D$ ) real bosonic and four real fermionic degrees of freedom. It is reminiscent of the unitary gauge, in which the unphysical components of $V$ are 'gauged away'. We should remark however, that this 
gauge choice is not invariant under SUSY transformations. Nevertheless, by a proper choice of a new gauge transformation the Wess-Zumino gauge can be restored.

Actually, the transformation rule in eq.(2.58) is sufficient for an Abelian gauge group (when all SF commute). For the non-Abelian group, it turns that to restore gauge invariance of the kinetic terms of chiral scalars and fermions the definition of the gauge transformation of the VSF has to be changed to

$$
e^{V} \rightarrow e^{-i \Lambda^{\dagger}(x)} e^{V} e^{i \Lambda(x)}
$$

Here $V \equiv V^{a} T^{a}$ and $\Lambda \equiv \Lambda^{a} T^{a}$ with the sum over the gauge indices $a$ is understood. Of course, in the Abelian case, where all superfields commute, this is equivalent to eq.(2.58).

\subsection{Towards the supersymmetric Lagrangian}

When we discussed scalar and vector superfields, the crucial observation was that the $F$-terms of the LcSF's and their products (i.e. the $\theta \theta$ components) and the D-terms of the VSF's (the $\theta \theta \bar{\theta} \bar{\theta}$ components) under SUSY transform into themselves and a total derivative. Thus if we define a Lagrangian as a sum of $F$ - and $D$-terms,

$$
\mathcal{L}=\mathcal{L}_{F}+\mathcal{L}_{D}
$$

the action $\int d^{4} x \mathcal{L}$ will remain invariant under SUSY transformations. Before we formulate the supersymmetric extension of the Standard Model in the next chapter, we will consider toy models with a single chiral matter superfield, a vector field and combining the gauge and matter superfields.

\subsection{The Wess-Zumino model of one chiral superfield}

Let us consider a single left-chiral superfield

$$
\Phi=\phi+\sqrt{2} \theta \psi-\theta \theta F .
$$

Using the identity $\theta \zeta \theta \xi=\frac{1}{2} \theta \theta \zeta \xi$ it is easy to calculate the $F$-terms of the products of $\Phi$,

$$
\begin{aligned}
& {[\Phi \Phi]_{F}=-2 \phi F-\psi \psi,} \\
& {[\Phi \Phi \Phi]_{F}=-3 \phi \phi F-2 \phi \psi \psi .}
\end{aligned}
$$

The first line contains a mass term for the Weyl spinor $\psi \psi$, while the second a Yukawa coupling of a scalar to a fermion $\phi \psi \psi$. The $F$-terms of the higher powers of $\Phi$ would be of the mass dimension $>4$, and thus non-renormalizable. Restricting to the renormalizable case, we can define the superpotential $W(\Phi)$ as a polynomial in $\Phi$ of degree at most 3,

$$
W(\Phi)=a \Phi+\frac{1}{2} m \Phi \Phi+\frac{1}{3 !} y \Phi \Phi \Phi
$$

where the parameters are of mass dimension $[a]=2,[m]=1$ and $[y]=0$. The resulting Lagrangian has the form

$$
\begin{aligned}
\mathcal{L}_{W Z, F} & =\int d^{2} \theta W(\Phi)+\int d^{2} \bar{\theta}(W(\Phi))^{\dagger} \equiv[W(\Phi)]_{F}+\text { h.c. } \\
& =-a F-m \phi F-\frac{m}{2} \psi \psi-\frac{y}{2} \phi \phi F-\frac{y}{2} \phi \psi \psi+h . c
\end{aligned}
$$


and contains the desired terms of a massive Weyl fermion coupled to a massive scalar. Observe that the fermion and the scalar appear in eq.(2.64) with the same mass $m$, which is a consequence of SUSY. Another consequence is that we cannot include in the superpotential a term of the form $\Phi^{\dagger} \Phi$ since its $\theta \theta$ component does not transform into itself plus a total derivative. In other words, the superpotential has to be a holomorphic function of the LcSF, i.e. it may depend only on $\Phi$ but not on $\Phi^{\dagger}$. If we remember that in the Standard Model masses of all fermions are generated by the Yukawa terms that involve a Higgs and its Hermitian conjugate, then we see the origin why in the supersymmetric extension we will have to employ two doublets of Higgses (with opposite hypercharges) to give masses to up- and down-type quarks.

So far so good, but we also need a dynamical part that would contain kinetic terms as well. However, since the $\Phi^{\dagger} \Phi$ term is a vector superfield, $\left(\Phi^{\dagger} \Phi\right)^{\dagger}=\Phi^{\dagger} \Phi$, its $D$-term can be used. Defining a Kähler potential $K=K\left(\Phi^{\dagger}, \Phi\right)$ as $K=\Phi^{\dagger} \Phi$, we find

$$
\mathcal{L}_{D, \mathrm{WZ}}=\int d^{4} \theta K=F^{\dagger} F+\left(\partial_{\mu} \phi\right)\left(\partial^{\mu} \phi\right)^{\dagger}+\frac{i}{2} \psi \sigma^{\mu}\left(\partial_{\mu} \bar{\psi}\right)-\frac{i}{2}\left(\partial_{\mu} \psi\right) \sigma^{\mu} \bar{\psi}
$$

As wanted, the $D$-term gives rise to canonical kinetic terms of the $\phi$ and the $\psi$ component fields. (A more general Kähler potential leads to more complicated terms in the Lagrangian, and at a quantum level it contains information on the wave-function renormalization).

However, the above formula does not contain a kinetic term for the $F$ component field. Therefore the equation of motion for $F$ (and $F^{\dagger}$ ) reduces to an algebraic equation

$$
0=\partial_{\mu} \frac{\partial \mathcal{L}}{\partial\left(\partial_{\mu} F\right)}-\frac{\partial \mathcal{L}}{\partial F}=-\frac{\partial \mathcal{L}}{\partial F}=-F^{\dagger}+a+m \phi+\frac{y}{2} \phi \phi
$$

which can be solved

$$
F^{\dagger}=-a-m \phi-\frac{y}{2} \phi \phi \equiv-\frac{\partial W(\phi)}{\partial \phi}
$$

and the $F$ and $F^{\dagger}$ components eliminated from the Lagrangian. This is why the $F$ component field is called an auxiliary and there are no particles associated with this field. The terms containing $F$ and $F^{\dagger}$ in eqs.(2.64) and (2.65) then read

$$
F^{\dagger} F-\left(a F+m \phi F+\frac{y}{2} \phi \phi F+\text { h.c. }\right)=-\left|a+m \phi+\frac{y}{2} \phi \phi\right|^{2} \equiv-\left|\frac{\partial W(\phi)}{\partial \phi}\right|^{2}
$$

In the above equations $W(\phi)$ is treated as a function of the scalar component field $\phi$ only, rather than the full superfield $\Phi$. In practice it is more useful for writing a Lagrangian in terms of component fields. Since the $a$ term can be eliminated by a simple shift of variables, we set $a=0$ and write the Lagrangian in the full glory

$$
\begin{aligned}
\mathcal{L}_{\mathrm{WZ}} & =\left(\partial_{\mu} \phi\right)\left(\partial^{\mu} \phi\right)^{\dagger}+\frac{i}{2} \psi \sigma^{\mu}\left(\partial_{\mu} \bar{\psi}\right)-\frac{i}{2}\left(\partial_{\mu} \psi\right) \sigma^{\mu} \bar{\psi} \\
& -|M|^{2} \phi \phi^{\dagger}-\frac{|y|^{2}}{4} \phi \phi \phi^{\dagger} \phi^{\dagger}-\left(\frac{m}{2} \psi \psi+\frac{m^{*} y}{2} \phi \phi \phi^{\dagger}+\frac{y}{2} \phi \psi \psi+\text { h.c. }\right) .
\end{aligned}
$$

It describes a system of spin 0 and spin 1/2 particles and three-point and four-point interactions between scalars and scalar-fermion-fermion Yukawa interactions. The masses of scalars and fermions and coupling strengths are all related and fully determined by the superpotential. 
Let us count degrees of freedom of component fields of the chiral supermultiplet. Before eliminating the $F$ component field, i.e. off-shell, we have two complex scalars $\phi$ and $F$, and therefore 4 bosonic degrees, and a Weyl spinor with 4 fermionic degrees. When the equation of motion for the $F$ component is used, i.e. on-shell, we have two bosonic $\phi$ and two fermionic $\psi$ degrees of freedom, so again the number of bosonic and fermionic degrees of freedom match.

The above argument can easily be generalized to a case of several left-chiral superfields $\Phi_{i}$. The most general superpotential takes the form

$$
W\left(\Phi_{i}\right) \equiv a_{i} \Phi_{i}+\frac{1}{2} m_{i j} \Phi_{i} \Phi_{j}+\frac{1}{3 !} y_{i j k} \Phi_{i} \Phi_{j} \Phi_{k},
$$

where the sum $\sum_{i j k}$ over all possible combinations of LcSF is understood and $a_{i}, m_{i j}$ and $y_{i j k}$ are constants. The resulting Lagrangian reads

$$
\begin{aligned}
\mathcal{L}_{\mathrm{WZ}} & =\left(\partial_{\mu} \phi_{i}\right)\left(\partial^{\mu} \phi_{i}\right)^{\dagger}+\frac{i}{2} \psi_{i} \sigma^{\mu}\left(\partial_{\mu} \bar{\psi}_{i}\right)-\frac{i}{2}\left(\partial_{\mu} \psi_{i}\right) \sigma^{\mu} \bar{\psi}_{i} \\
& -\sum_{i}\left|\frac{\partial W}{\partial \phi_{i}}\right|^{2}-\frac{1}{2} \frac{\partial^{2} W}{\partial \phi_{i} \partial \phi_{j}} \psi_{i} \psi_{j}-\frac{1}{2} \frac{\partial^{2} W^{\dagger}}{\partial \phi_{i}^{\dagger} \partial \phi_{j}^{\dagger}} \bar{\psi}_{i} \bar{\psi}_{j}
\end{aligned}
$$

\subsection{Non-renormalization of the superpotential}

Let us come back to the point that we started with, namely the absence of quadratically divergent radiative correction to the scalar mass. To simplify things consider the case of a single superfield, eq.(2.69). First, notice that there are no diagrams that would contribute to the fermion mass correction; simply, the arrows in the the loops cannot match appropriately. For the one-loop correction to the scalar mass there are two diagrams: with the scalar circulating in the loop coupled via the quartic scalar coupling, and with the fermion coupled by the Yukawas. However, since the masses and couplings are correlated, the two diagrams cancel completely. In this way supersymmetry does indeed control radiative corrections to the scalar masses.

In fact, it can be shown that not only the mass term but the whole superpotential $W$ receives no additive radiative corrections in any order of perturbation theory [9]. Following Shirman [10], here we give the argument due to Seiberg [11]. It exploits the symmetries and holomorphy of superpotential. To this end we define a $U(1)_{R}$-symmetry that acts on superspace coordinates $\theta$ and $\bar{\theta}, \theta \rightarrow e^{i \alpha} \theta$ and $\bar{\theta} \rightarrow e^{-i \alpha} \bar{\theta}$. If the R-charge of $\theta$ is taken $R_{\theta}=1$, different components of the superfields transform differently under $R$-symmetry. For example, assigning an $R$-charge $R$ to the chiral superfield $\Phi$ in eq.(2.62), its lowest component $\phi$ has the same $R$-charge as the superfield itself, its $\theta$ component $\psi$ has $R$-charge $R-1$ and its $\theta^{2}$ component $F$ has $R$-charge $R-2$.

From the integration rules eq.(2.33) it follows that $R_{d \theta}=-1$. As a result, kinetic terms of the Lagrangian arising the from Kähler potential are always invariant under the $R$-symmetry since $\Phi^{\dagger} \Phi$ and $d^{4} \theta$ are real. On the other hand, the full Lagrangian is invariant under $R$-symmetry only if the superpotential transforms with the charge $2, W \rightarrow e^{2 i \alpha} W$. Imposing such a requirement on the superpotential determines $R$-symmetry charges of the superfields. However, a consistent assignment of $R$-charges may not exist and then $R$-symmetry is explicitly broken by some terms in the Lagrangian. For example, in a massless Wess-Zumino model of eq.(2.63) with $a=m=0$, assigning an $R$-charge of $2 / 3$ to $\Phi$ gives the Lagrangian invariant under $R$-symmetry, while with $m \neq 0$ there is no charge assignment which leaves the Lagrangian invariant. 
To prove the non-renormalization theorem we promote the superpotential parameters to background superfields [11]. The model of eq.(2.63) (with $a=0$, since as we said, $a$ can be eliminated by a shift of variables) is considered as an effective low-energy description of a more fundamental theory in which parameters $m$ and $y$ arise as vacuum expectation values of the lowest components of heavy superfields (spurions). As a result, with the charges of the dynamical superfield $\Phi$ and spurions as follows

$\begin{array}{ccc} & U(1)_{R} & U(1) \\ \Phi & 1 & 1 \\ m & 0 & -2 \\ \lambda & -1 & -3\end{array}$

the theory has a $U(1) \times U(1)_{R}$ global symmetry (spontaneously broken by expectation values of the spurions $m$ and $y$ ).

In this approach superpotential must be described by a holomorphic function of both the dynamical and background superfields $W(\Phi, m, y)$. To have the correct transformation properties under global symmetries, its form is restricted to be

$$
W=\frac{m}{2} \Phi^{2} f\left(\frac{y \Phi}{m}\right)
$$

In the weak coupling limit the effective superpotential should approach the classical one and therefore there should exist a Taylor series expansion of $f$ in $y \Phi / m$ :

$$
W=\frac{m}{2} \Phi^{2}\left(1+\frac{2}{3 !} \frac{y \Phi}{m}+\mathcal{O}\left(\frac{y^{2} \Phi^{2}}{m^{2}}\right)\right)=\frac{m}{2} \Phi^{2}+\frac{y}{3 !} \Phi^{3}+\mathcal{O}\left(y^{2}\right)
$$

Thus

$$
f\left(\frac{y \Phi}{m}\right)=1+\frac{2}{3 !} \frac{y \Phi}{m}+\mathcal{O}\left(y^{2}\right)
$$

Furthermore, the $m \rightarrow 0$ limit must be regular, and therefore $W$ should not contain negative powers of $m$. Thus (2.63) is exact [11]. No higher dimension terms are generated. This, in particular, means that there are no counterterms leading to renormalization of $m$ or $y$.

\subsection{The gauge sector}

The product of the VSF is also a VSF. However, its $D$-term, although supersymmetric, does not provide a kinetic term for the corresponding spin 1 vector field $v_{\mu}$ in the Lagrangian. Let us begin with the Abelian gauge symmetry. To write down kinetic terms for the vector superfields we define a chiral spinor superfield

$$
\mathcal{W}_{\alpha} \equiv-\frac{1}{4}(\bar{D} \bar{D}) D_{\alpha} V, \quad \overline{\mathcal{W}}_{\dot{\alpha}} \equiv-\frac{1}{4}(D D) \bar{D}_{\dot{\alpha}} V .
$$

$\mathcal{W}_{\alpha}$ is a LcSF because $\bar{D}_{\dot{\alpha}} \bar{D} \bar{D}=0$, which implies $\bar{D}_{\dot{\alpha}} \mathcal{W}_{\alpha}=0$. Correspondingly, $\overline{\mathcal{W}}_{\dot{\alpha}}$ is a RcSF. In terms of component fields it reads (we refer to [5] for details of the calculations)

$$
\mathcal{W}_{\alpha}=-i \lambda_{\alpha}-\theta \theta \sigma_{\alpha \dot{\beta}}^{\nu} \partial_{\nu} \bar{\lambda}^{\dot{\beta}}-\frac{i}{2} \theta_{\beta}\left(\sigma^{\mu} \bar{\sigma}^{\nu}\right)_{\alpha}^{\beta} F_{\mu \nu}+\theta_{\alpha} D
$$


where $F_{\mu \nu} \equiv \partial_{\mu} v_{\nu}-\partial_{\nu} v_{\mu}$ is the usual field strength tensor and the component fields are functions of $y^{\mu}=x^{\mu}-i \theta \sigma^{\mu} \bar{\theta}$. The lowest component field of $\mathcal{W}_{\alpha}$ is a spinor, giving the name to this LcSF. Note that $\mathcal{W}_{\alpha}$ and $\overline{\mathcal{W}}_{\dot{\alpha}}$ are gauge independent, as suggested by the presence of the field strength tensor. This can be verified by using the definition of $\mathcal{W}_{\alpha}$ and gauge transformation, eq.(2.58) and using $\bar{D}_{\dot{\alpha}} \Lambda=0$. From the spinor LcSF (and RcSF) we can form the Lorentz scalar by contracting the spinor indices and find that, indeed, the $F$-terms $\left[\mathcal{W}^{\alpha} \mathcal{W}_{\alpha}\right]_{\theta \theta}+\left[\overline{\mathcal{W}}_{\dot{\alpha}} \overline{\mathcal{W}}^{\dot{\alpha}}\right]_{\bar{\theta} \bar{\theta}}$ do contain the desired kinetic terms

$$
\frac{1}{4}\left[\mathcal{W}^{\alpha} \mathcal{W}_{\alpha}\right]_{\theta \theta}+\frac{1}{4}\left[\overline{\mathcal{W}}_{\dot{\alpha}} \overline{\mathcal{W}}^{\dot{\alpha}}\right]_{\bar{\theta} \bar{\theta}}=-\frac{1}{4} F^{\mu \nu} F_{\mu \nu}-\frac{i}{2}\left(\partial_{\mu} \lambda\right) \sigma^{\mu} \bar{\lambda}+\frac{i}{2} \lambda \sigma^{\mu}\left(\partial_{\mu} \bar{\lambda}\right)+\frac{1}{2} D^{2}
$$

for the gauge boson field $v_{\mu}$ and its fermionic partner $\lambda$.

We already mentioned that the $R$-charges of matter fields may depend on the model under consideration. In contrast, the $R$-charges of the fields in a vector multiplet are uniquely fixed. Indeed $\mathcal{W}^{\alpha} \mathcal{W}_{\alpha}$ has $R$-charge 2 . Since gaugino is the lowest component of $\mathcal{W}^{\alpha}$ its $R$-charge is 1 while the $D$-term and the gauge field $v_{\mu}$ are neutral (as expected for real fields).

Since there is no kinetic term for $D$ in eq.(2.77), the $D$ component field is an auxiliary, like the $F$ component field of the LcSF, and can be eliminated using the equation of motion. First, however, we need to find all other terms containing $D$. For an Abelian gauge field, the $D$ component field of $V$ under gauge transformation obtains a total derivative, as can be seen by inspecting eq.(2.58), and therefore we can add to the Lagrangian a term

$$
\mathcal{L}_{\mathrm{FI}}=2[\xi V]_{\theta \theta \bar{\theta} \bar{\theta}}=\xi D,
$$

where $\xi$ is a constant with mass dimension $[\xi]=2$ and the factor 2 is added for convenience. Such a term is called a Fayet-Iliopoulos term [12] and is important when spontaneous breaking of SUSY is considered. For the non-Abelian gauge group the FI term is forbidden since the VSF $V^{a}$, and the $D^{a}$ component field, carries a group index $a$. Nevertheless, when we couple the gauge sector to the matter superfields, other terms containing $D$ will appear.

Since these lectures are addressed mainly to the experimentalists attending the school, we refrain from going into details and simply state the results.

For the non-Abelian gauge group we have already introduced the compact notation $V \equiv V^{a} T^{a}$ for the gauge VSF, where $T^{a}$ are the generators in the adjoint representation. We also introduce the short-hand notation for the spinor chiral superfields $\mathcal{W}_{\alpha} \equiv \mathcal{W}_{\alpha}^{a} T^{a}$ and $\overline{\mathcal{W}}_{\alpha} \equiv \overline{\mathcal{W}}_{\dot{\alpha}}^{a} T^{a}$. Their definition has to be modified to

$$
\mathcal{W}_{\alpha}=-\frac{1}{8 g} \bar{D} \bar{D} e^{-2 g V} D_{\alpha} e^{2 g V}, \quad \overline{\mathcal{W}}_{\dot{\alpha}} \equiv \frac{1}{8 g} D D e^{2 g V} \bar{D}_{\dot{\alpha}} e^{-2 g V},
$$

where $g$ is the gauge coupling constant. In terms of the component fields $\mathcal{W}_{\alpha}^{a}$ reads

$$
\mathcal{W}_{\alpha}^{a}=-\frac{i}{2} \theta_{\beta}\left(\sigma^{\mu} \bar{\sigma}^{\nu}\right)_{\alpha}^{\beta} F_{\mu \nu}^{a}-\theta \theta \sigma_{\alpha \dot{\alpha}}^{\mu}\left(D_{\mu} \bar{\lambda}^{a}\right)^{\dot{\alpha}}-i \lambda_{\alpha}^{a}+\theta_{\alpha} D^{a},
$$

where the field-strength tensor and the (gauge) covariant derivatives are given by

$$
F_{\mu \nu}^{a}=\partial_{\mu} v_{\nu}^{a}-\partial_{\nu} v_{\mu}^{a}-g f^{a b c} v_{\mu}^{b} v_{\nu}^{c}, \quad\left(D^{\mu} \bar{\lambda}^{a}\right)^{\dot{\alpha}}=\left(\partial^{\mu} \bar{\lambda}^{a}\right)^{\dot{\alpha}}-g f^{a b c}\left(v^{b}\right)^{\mu}\left(\bar{\lambda}^{c}\right)^{\dot{\alpha}}
$$

and the component fields are functions of $y^{\mu}=x^{\mu}-i \theta \sigma^{\mu} \bar{\theta}$. 
The above expressions make it explicit that $\mathcal{W}_{\alpha}$ (and $\overline{\mathcal{W}}_{\dot{\alpha}}$ ) is not any more gauge invariant under non-Abelian transformations. In fact they transform as

$$
\mathcal{W}_{\alpha} \rightarrow e^{-2 i g \Lambda} \mathcal{W}_{\alpha} e^{2 i g \Lambda}, \quad \overline{\mathcal{W}}_{\dot{\alpha}} \rightarrow e^{-2 i g \Lambda^{\dagger}} \overline{\mathcal{W}}_{\dot{\alpha}} e^{2 i g \Lambda^{\dagger}},
$$

which is analogous to the non-susy case. Therefore the trace over the group indices $\operatorname{Tr} \mathcal{W}^{\alpha} \mathcal{W}_{\alpha}=$ $\frac{1}{2} \mathcal{W}^{a \alpha} \mathcal{W}_{\alpha}^{a}$ is gauge and Lorentz invariant, and its $\theta \theta$ component SUSY invariant. Therefore in the non-Abelian case the Lagrangian for the pure gauge sector takes the form

$$
\mathcal{L}_{\text {gauge }}=\frac{1}{4}\left[\mathcal{W}^{a \alpha} \mathcal{W}_{\alpha}^{a}\right]_{\theta \theta}+\frac{1}{4}\left[\overline{\mathcal{W}}_{\dot{\alpha}}^{a} \overline{\mathcal{W}}^{a \dot{\alpha}}\right]_{\bar{\theta} \bar{\theta}}
$$

Let us now add a set of $\operatorname{LcSF} \Phi_{i}$ that transform under a certain representation of the gauge group as $\Phi \rightarrow \Phi^{\prime}=e^{2 i g \Lambda} \Phi$, or more explicitly

$$
\Phi_{i} \rightarrow \Phi_{i}^{\prime}=\left(e^{-2 i g \Lambda^{a} T^{a}}\right)_{i j} \Phi_{j}
$$

with the generators $T^{a}$ in the corresponding representation. For the Hermitian conjugate SF we have $\Phi^{\dagger} \rightarrow \Phi^{\prime \dagger}=\Phi^{\dagger} e^{2 i g \Lambda^{\dagger}}$. Since $\Lambda$ is a set of LcSF, $\Lambda^{\dagger}$ is a RcSF, and the Kähler potential transforms non-trivially

$$
\Phi^{\dagger} \Phi \rightarrow \Phi^{\prime \dagger} \Phi^{\prime}=\Phi^{\dagger} e^{2 i g \Lambda^{\dagger}(x)} e^{-2 i g \Lambda(x)} \Phi \neq \Phi^{\dagger} \Phi
$$

because for a local transformation $\Lambda(x) \neq \Lambda^{\dagger}(x)$. The remedy is to redefine the kinetic part for the chiral superfields as

$$
\Phi^{\dagger} \Phi \rightarrow \Phi^{\dagger} e^{2 g V} \Phi
$$

where the group indices are understood. Then the Lagrangian reads

$$
\mathcal{L}=\frac{1}{4}\left[\mathcal{W}^{a} \mathcal{W}^{a}\right]_{\theta \theta}+\frac{1}{4}\left[\overline{\mathcal{W}}^{a} \overline{\mathcal{W}}^{a}\right]_{\bar{\theta} \bar{\theta}}+\left[\Phi^{\dagger} e^{2 g V} \Phi\right]_{\theta \theta \bar{\theta} \bar{\theta}}+[W(\Phi)]_{\theta \theta}+\left[W^{\dagger}\left(\Phi^{\dagger}\right)\right]_{\bar{\theta} \bar{\theta}}
$$

In terms of the component fields for the chiral superfields $\Phi_{i}$ transforming under the gauge group with the gauge vector superfields $V_{\mathrm{WZ}}^{a}$ for a general gauge group with the generators $T_{i j}^{a}$ satisfying $\left[T^{a}, T^{b}\right]=i f^{a b c} T^{c}$ algebra, it takes the form

$$
\begin{aligned}
\mathcal{L} & =\left(D_{\mu} \phi\right)_{i}^{\dagger}\left(D^{\mu} \phi\right)_{i}+\frac{i}{2} \psi_{i} \sigma^{\mu}\left(D_{\mu} \bar{\psi}\right)_{i}-\frac{i}{2}\left(D_{\mu} \psi\right)_{i} \sigma^{\mu} \bar{\psi}_{i} \\
& -\frac{1}{4} F_{\mu \nu}^{a}\left(F^{a}\right)^{\mu \nu}+\frac{i}{2} \lambda^{a} \sigma^{\mu}\left(D_{\mu} \bar{\lambda}\right)^{a}-\frac{i}{2}\left(D_{\mu} \lambda\right)^{a} \sigma^{\mu} \bar{\lambda}^{a} \\
& -\sqrt{2} i g \bar{\psi}_{i} \bar{\lambda}^{a} T_{i j}^{a} \varphi_{j}+\sqrt{2} i g \phi_{i}^{\dagger} T_{i j}^{a} \psi_{j} \lambda^{a} \\
& -\frac{1}{2} \frac{\partial^{2} W}{\partial \phi_{i} \partial \phi_{j}} \psi_{i} \psi_{j}-\frac{1}{2} \frac{\partial^{2} W^{\dagger}}{\partial \phi_{i}^{\dagger} \partial \phi_{j}^{\dagger}} \bar{\psi}_{i} \bar{\psi}_{j}-V\left(\phi_{i}, \phi_{j}^{\dagger}\right)
\end{aligned}
$$

The scalar potential is the sum of the $F$-terms and $D$-terms and reads

$$
V\left(\phi_{i}, \phi_{j}^{\dagger}\right)=F_{i}^{\dagger} F_{i}+\frac{1}{2}\left(D^{a}\right)^{2}=\sum_{i}\left|\frac{\partial W}{\partial \phi_{i}}\right|^{2}+\frac{1}{2} \sum_{a}\left(g \phi_{i}^{\dagger} T_{i j}^{a} \phi_{j}+\xi^{a}\right)^{2} .
$$

The Fayet-Iliopoulos term $\mathcal{L}_{\mathrm{FI}}=2 \sum_{a} \xi^{a}\left[V^{a}\right]_{\theta \theta \bar{\theta} \bar{\theta}}$ can be present only for $U(1)$ gauge factors, and $W$ is the most general superpotential consistent with the assumed gauge symmetry. 


\section{The Minimal supersymmetric extension of the Standard Model}

\subsection{Particle content}

The minimally supersymmetrized SM is uniquely defined: the particle content and couplings are fixed with no new parameters introduced. Each SM particle $p$ has a partner with the same quantum numbers but with spin differing by $1 / 2$ - called a spartner and denoted by a tilde over the symbol $\tilde{p}$. Following a common convention of considering the left-chiral Weyl fermions as basic particles, the left-chiral quarks and leptons (doublets under $\left.S U(2)_{L}\right)$ and left-chiral antiquarks and antileptons (singlets under $\left.S U(2)_{L}\right)$ are assigned to be fermions in the corresponding leftchiral superfields $Q, L$ and $U^{c}, E^{c}$, respectively. There are three copies of the quark and lepton superfields, one for each generation. The right-chiral fermions are the conjugates of the left-chiral antifermions. Here the chirality refers to the transformation properties under the weak $S U(2)_{L}$ gauge group. For a massless fermion chirality is equivalent to helicity, while for the spinless sfermions such a parallel does not exist.

The gauge bosons are placed in the vector supermultiplet and each one is accompanied by a spin $1 / 2$ Weyl fermion called a gaugino. Thus we have gluinos, winos and a bino as superpartners of the gluons and electroweak $W$ and $B$ gauge bosons.

We already mentioned that in the supersymmetric version we will need two doublets of the Higgs fields to give masses to both up- and down-type fermions. However, there is another argument why we need an even number of Higgs doublets which is related to the axial vector anomaly cancelation. In the SM the anomaly cancels non-trivially between the quarks and leptons. When we promote a Higgs field to a superfield it comes with its fermion partner (higgsino). Each higgsino makes a non-zero contribution to this anomaly. These contributions cancel if we include pairs of higgsinos with opposite hypercharges. The minimal supersymmetric standard model (MSSM) employs two Higgs doublet superfields $H_{1}, H_{2}$ with hypercharges $-1 / 2,+1 / 2$, respectively, containing scalar Higgses accompanied by spin $1 / 2$ higgsinos $\tilde{H}_{1}, \tilde{H}_{2}$.

\subsection{Construction of the MSSM Lagrangian}

Once the choice of the gauge group $S U(3)_{c} \times S U(2)_{L} \times U(1)_{Y}$ and the content of the matter chiral fields with definite quantum numbers has been made, the kinetic terms and the gauge couplings of the MSSM Lagrangian are fully determined by supersymmetry. The Lagrangian is a sum of terms of the form eq.(2.87). The only parameters that need to be introduced are the gauge couplings $g_{1}, g_{2}$ and $g_{3}$. Also the superpotential $W$ has to be specified. Since it provides the source for non-linear scalar-fermion-fermion couplings, we should include the appropriate terms that provide the right Yukawa couplings to generate fermion masses via the spontaneous gauge symmetry breaking. The superpotential has to bo holomorphic in LcSF.

The right choice is

$$
W=h_{E} H_{1} L E^{c}+h_{D} H_{1} Q D^{c}+h_{U} H_{2} Q U^{c}-\mu H_{1} H_{2}
$$

where color, $\mathrm{SU}(2)$ and generation indices are suppressed. Here $L, E^{c}, Q, D^{c}, U^{c}, H_{i}$ denote leftchiral superfields with self-obvious (s)particle content, $h_{i}$ are the corresponding Yukawa couplings (matrices in the generation space). Apart from the trilinear terms with $h_{i}$, the term with dimensionfull Higgs(ino) mass parameter $\mu$ is gauge invariant and can be included in $W$. Actually this is the 
only parameter of mass dimension 1 . Since this is in the superpotential, it does not receive additive radiative corrections. If it is set to zero, a higgsino state would be massless and should have been already detected. So it is needed for phenomenological reasons.

In principle the superpotential can contain other terms

$$
W_{\not R}=\lambda_{a b d} L_{a} L_{b} E_{d}^{c}+\lambda_{a b d}^{\prime} L_{a} Q_{b} D_{d}^{c}+\epsilon_{a} L_{a} H_{2}+\lambda_{a b d}^{\prime \prime} U_{a}^{c} D_{b}^{c} D_{d}^{c}
$$

with the generation indices $a, b, d$ explicitly written. The first three terms generate lepton-number (L) and the last term baryon-number (B) violating interactions in the Lagrangian. In SM such interactions are forbidden by Lorentz invariance and particle content. In the SUSY version with scalar superpartners, such interactions are fully consistent with all symmetries. Phenomenologically the presence of both L- and B-number violating interactions is disastrous since it leads to fast proton decay. The simplest, and most popular, solution is to suppress all $W_{\not R}$ terms by imposing a symmetry, called $R$-parity [13], defined as

$$
R_{p}=(-1)^{3 B-L+2 s}
$$

Imposing $R$-parity has important consequences: sparticles are created in pairs in particle collisions, among the decay products of a sparticle there is always a sparticle, and the lightest sparticle (LSP) is stable. This makes the LSP, in many SUSY models the lightest neutralino, an attractive candidate for the dark matter particle - which is one of the most welcome features of the supersymmetric extension of the SM.

Negative results of SUSY searches indicate that sparticles cannot be degenerate in mass with corresponding particles and supersymmetry must be broken. Since no viable model of SUSY breaking within the MSSM itself can be constructed, the most popular scenario is to invoke the socalled hidden sector where spontaneous supersymmetry breaking occurs and with the help of some messenger fields it is mediated to the visible sector generating in the MSSM Lagrangian terms that break SUSY explicitly. To maintain the cancelation of quadratic divergencies needed for solving the hierarchy problem, SUSY breaking terms must be soft, i.e. of dimension less than 4 . The most general form of soft terms includes gaugino $\left(\tilde{\lambda}_{a}\right)$ and scalar $\left(\phi_{i}\right)$ masses $\left(M_{a}, M_{i j}^{2}\right)$, and scalar bilinear $\left(b_{i j}\right)$ and trilinear $\left(A_{i j k}\right)$ couplings [14]

$$
-\mathcal{L}_{\text {soft }}=\frac{1}{2} M_{a}^{2} \tilde{\lambda}_{a} \tilde{\lambda}_{a}+M_{i j}^{2} \phi_{i}^{\dagger} \phi_{j}+b_{i j} \phi_{i} \phi_{j}+A_{i j k} \phi_{i} \phi_{j} \phi_{k}+h . c .
$$

Before SUSY breaking terms have been introduced, the supersymmetric Lagrangian contained as parameters only the gauge and Yukawa couplings and the $\mu$ mass parameter. Adding $\mathcal{L}_{\text {soft }}$ brings in 105 new parameters The unconstrained MSSM (uMSSM) is usually understood as an effective low-energy model at a $\mathrm{TeV}$ scale defined by three assumptions: a) minimal particle content, b) $R$-parity conservation, c) most general soft-supersymmetry breaking terms. The number of parameters could be further enlarged by relaxing a) and/or b), or reduced by constraining c) with additional assumptions on SUSY breaking mechanism.

After the gauge symmetry breaking, the superpotential generates terms that mix the electroweak gauginos and higgsinos giving rise to mass eigenstates: two charged (charginos $\tilde{\chi}_{1,2}^{ \pm}$) and four neutral (neutralinos $\tilde{\chi}_{1,2,3,4}^{0}$ ). The particle content of the MSSM is illustrated in fig.1. For the complete list of Feynman rules of the MSSM we refer to Rosiek [15] 


\section{MSSM: particles and sparticles}

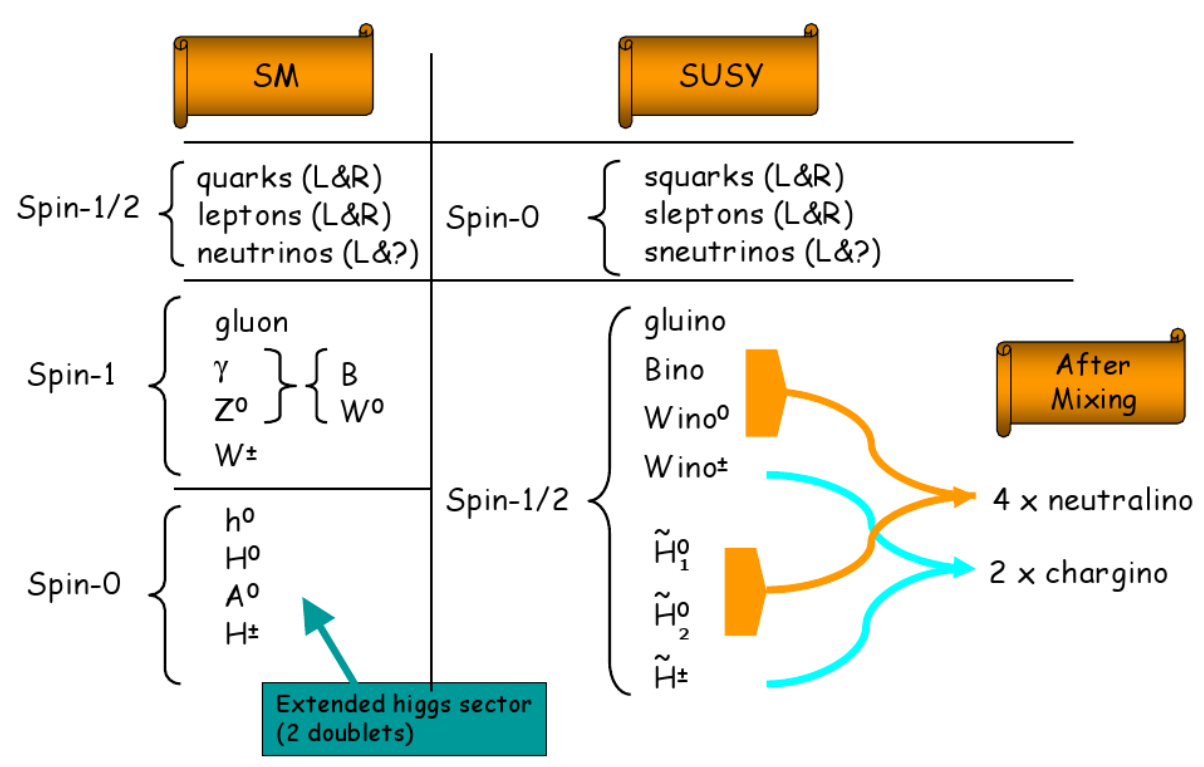

Figure 1: Particle and sparticle content of the MSSM

As the soft SUSY breaking is explicit, the Appelquist-Carazzone theorem [16] applies to the superpartner spectrum. Thus, SUSY virtual effects disappear at least as an inverse of the SUSY breaking scale, $\mathcal{O}\left(1 / M_{S U S Y}\right)$, and can naturally be arranged compatible with the electroweak (EW) precision data. It is nevertheless interesting to note that global fits to EW and DM data [17], at least in the constrained MSSM (defined below), usually referred to as mSUGRA, point to a rather low values of SUSY breaking parameters [18], which interestingly enough are close to the benchmark point SPS1a of [19]. Together with the strong indication for a light Higgs boson it fuels hopes for a discovery of the Higgs boson(s) and at least some of supersymmetric particles at the LHC.

However, with that many new parameters it is hard to accept the unconstrained MSSM as a fundamental theory. Moreover, in most of the 105-parameter space the model exhibits phenomenologically bad features, like unsuppressed FCNC and CP-violating phenomena, color or charge breaking vacua etc. The MSSM is viable only in some regions of the parameter space with a certain degree of universality.

Since the gauge coupling unification suggests that physics might be simpler at or near the unification scale, renormalization-group equations (RGE) can be used to provide the link between low- and high-scale theories. In the top-down approach a plethora of theoretical scenarios of hidden sectors and mediation of SUSY-breaking has been examined, like gravity-, gauge-, anomaly-, mixed-, ..., mediation. Then the RGE are used to derive the low-energy MSSM parameters. It turns out that phenomenological Lagrangian depends crucially only on gross features: which hiddensector fields develop the largest F- or D-term vacuum expectation values, what is the mediation mechanism, what are dominant effects producing hidden-visible sector couplings: at tree level, or loop-induced etc. As a result, each scenario can be characterized by a handful of independent 
parameters which makes the phenomenological analyses of low-energy theory much simpler and more predictive. For example, the mSUGRA scenario mentioned above is defined by universal scalar $\left(M_{0}\right)$ and gaugino $\left(M_{1 / 2}\right)$ masses and universal trilinear $\left(A_{0}\right)$ scalar couplings at some unification scale, while the universal bilinear parameter is traded for the ratio of the Higgs vacuum expectation values $\tan \beta=v_{2} / v_{1}$ from the condition of reproducing the correct mass of the $Z$ boson, and sign of the higgsino-mass parameter $\mu$.

However the top-down approach may be too restrictive: the phenomenologically viable region of the parameter space is larger than any RGE-derived region of the above scenarios. Moreover, our imagination of devising high-scale supersymmetry-breaking scenarios is certainly limited.

At present only the experimental limits on the parameter space can be used to gain some insight on the SUSY breaking. The non-discovery of SUSY and a light Higgs boson at LEP2, Tevatron and HERA puts the solution of the naturalness problem in a subtle position: fine tuning of order a few percent is required to reproduce the EW scale and evade experimental constraints. This problem, called the supersymmetric fine-tuning, has attracted much attention and is one of the main driving forces to go beyond the MSSM $^{2}$

Once supersymmetry is discovered, we will have to face the problem of reconstructing the lowenergy supersymmetry Lagrangian parameters from experimental measurements with minimum of theoretical assumptions. Only then in the bottom-up approach [20] we can attempt use the RGE as a telescope to explore the high-energy physics by exploiting the low-energy experimental input to the maximum extent possible.

\section{Phenomenology of SUSY at colliders ${ }^{3}$}

At present the most restrictive direct limits on the SUSY parameter space come from negative results of SUSY searches at two colliders: Tevatron at Fermilab and HERA at DESY. HERA already finished its operation in 2007, but Tevatron still collects new data. With the start of the LHC at the end of 2009 significant improvements, and hopefully many new discoveries can be expected in coming months and years.

\subsection{SUSY searches at Tevatron and HERA}

The Tevatron experiments have investigated various SUSY scenarios: a more or less constrained MSSM with a neutralino LSP assumed - both $R$-parity conserving and violating cases have been considered; gauge-mediated SUSY breaking (GMSB) with a gravitino LSP and a neutralino NLSP (next-to-lightest SUSY particle); anomaly-mediated SUSY breaking (AMSB) with a wino LSP and a long-lived chargino; split-SUSY with heavy scalars and a long-lived gluino. Because of lower kinematic reach, the HERA experiments concentrated on single sparticle production processes in the $R$-parity violated cases: light stop production in uMSSM; squark production in mSUGRA; gaugino production in UMSSM and GMSB. Below only selected results are presented; more results can be found in Ref.[21].

\footnotetext{
${ }^{2}$ The discussion of Beyond MSSM is beyond the scope of these lectures.

${ }^{3}$ This chapter is based on the updated version of my lectures given at the APCTP Summer Institute 2006 in Pohang, August 23-30, 2006, Korea, http://apctp.org/conferences/SI2006/index.htm.
} 

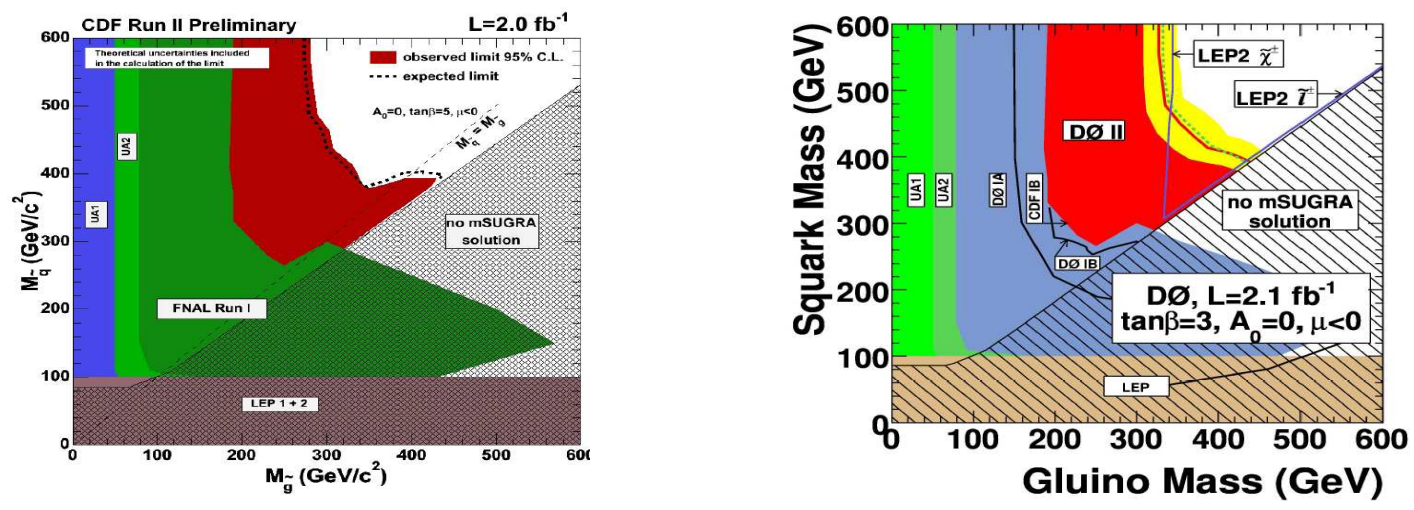

Figure 2: Exclusion plots in the plane of generic squark and gluino masses.

If squarks are lighter than gluinos, they are expected to decay according to $\tilde{q} \rightarrow q \tilde{\chi}_{1}^{0}$, while gluinos, if they are lighter that squarks, are expected to decay according to $\tilde{g} \rightarrow q \bar{q} \tilde{\chi}_{1}^{0}$. Pair production of light squarks leads to $\geq$ two jets; pair production of light gluinos leads to $\geq$ four jets; the associated production of a squark and a gluino of similar masses leads to $\geq$ three jets. Possible cascade decays, for example $\tilde{q} \rightarrow q^{\prime} \tilde{\chi}^{ \pm}$, complicate the picture and a specific model, such as mSUGRA, is needed to interpret the search results. Multi-jet events with fake missing $E_{T}$ due to jet energy mismeasurements and the associated production of $W+$ jets with missed lepton from $W \rightarrow \ell \nu$ constitute instrumental background, while irreducible comes from the associated production of $Z+$ jets with $Z \rightarrow \nu \bar{\nu}$. The generic search assumes a number of cuts on: minimum number of jets, missing $E_{T}$, and the sum of jet transverse energies, a veto on isolated leptons, and cuts on angles between the missing $E_{T}$ and jet directions. The inclusive search for squarks and gluinos has been performed by CDF and D0 with data samples of about $2 \mathrm{fb}^{-1}$. Since no excess is seen in data over expected background, exclusion domains in the plane of the squark and gluino masses have been computed, Fig. 2. Masses below < $280 \mathrm{GeV}$ (CDF), $<308 \mathrm{GeV}$ (D0) for gluino, and <380 $\mathrm{GeV}$ (CDF,D0) for squarks, are excluded [22].

Electron-proton collisions at HERA are well suited to the search for squarks, since such states can be produced by an appropriate coupling of the incoming lepton and a quark in the proton. Events with isolated high- $p_{T}$ leptons, jet(s) and missing energy, have been observed at HERA in $e^{+} p$ collisions, and created a lot of excitement. With the increased statistics no significant deviation from the SM has been observed, and exclusion limits were determined in the framework of the MSSM [23]. Nevertheless, some puzzling H1 events with isolated muons, not easily explained by the SM mechanisms, still invite speculations on their origin [24].

\subsection{Expectations at the LHC}

The strongly interacting squarks and gluinos ( $\tilde{q}$ and $\tilde{g})$, with masses in the $\mathrm{TeV}$ range, will be copiously produced at the LHC. Their production cross sections (typically in the picobarn range) are comparable to cross sections of jets with transverse momenta $p_{t} \sim$ SUSY masses. Rates of directly produced weakly interacting sparticles are much lower. Squarks and gluinos will promptly decay into jets and lighter SUSY particles which will further decay. Their decay chains are model dependent, but generically one can expect in the final state high- $p_{t}$ jets and leptons, possibly large 
missing energy $E_{t}$, or displaced vertices etc. Since the LHC detectors are designed to detect jets, isolated leptons and photons, displaced vertices, measure energies and transverse momenta and missing transverse energy, they are well equipped to cover a broad spectrum of possible decay modes of SUSY particles. There have been many experimental analyses demonstrating the capabilities of LHC detectors ATLAS and CMS [25, 26], to which we refer for details.

\subsubsection{Inclusive searches at LHC}

Sparticle production in $p p$ collisions at the LHC is dominated by $\tilde{q}$ and $\tilde{g}$. Leptonic decays may or may not be large but jets are always produced with transverse momenta $p_{t}$ of the order of sparticle masses. If the LSP is stable, as in scenarios with $R$-parity conserved, it will escape undetected giving large $E_{t}$. The SM background events from top quark, $W$ and $Z$ boson decays do not have such high- $p_{t}$ objects.

Motivated by these observations, a set of simple cuts can be designed to enhance the signal over the background in inclusive "transverse" searches for SUSY particles. For example, it has been demonstrated [25] that in typical mSUGRA scenarios, requiring at least four jets with large $p_{t}^{i}$ and large

$$
M_{\mathrm{eff}}=\sum_{i=1, \ldots 4} p_{t}^{i}+\not E_{t}
$$

and selecting events spherical in the transverse plane (where specific cuts on $E_{t}, p_{t}^{i}, M_{\text {eff }}$ and sphericity depend on details of the model) can be sufficient to discover new particles. To reduce the background further, hard, isolated lepton(s) may be required and their $p_{t}$ is then included in the definition of $M_{\text {eff. }}$. The reach of inclusive searches at $10^{-1} \mathrm{fb}$ is illustrated in Fig. 3; and squarks and gluinos with masses up to $\sim 2.5 \mathrm{TeV}$ can be found at LHC with $100 \mathrm{fb}^{-1}$. Monte Carlo studies have also shown that the position of the peak in $M_{\text {eff }}$ distribution correlates quite well with sparticle masses, namely $M_{\text {eff }} \sim \min \left(m_{\tilde{q}}, m_{\tilde{g}}\right)$, providing a first estimate of the overall SUSY mass scale, Fig. 3 right panel.
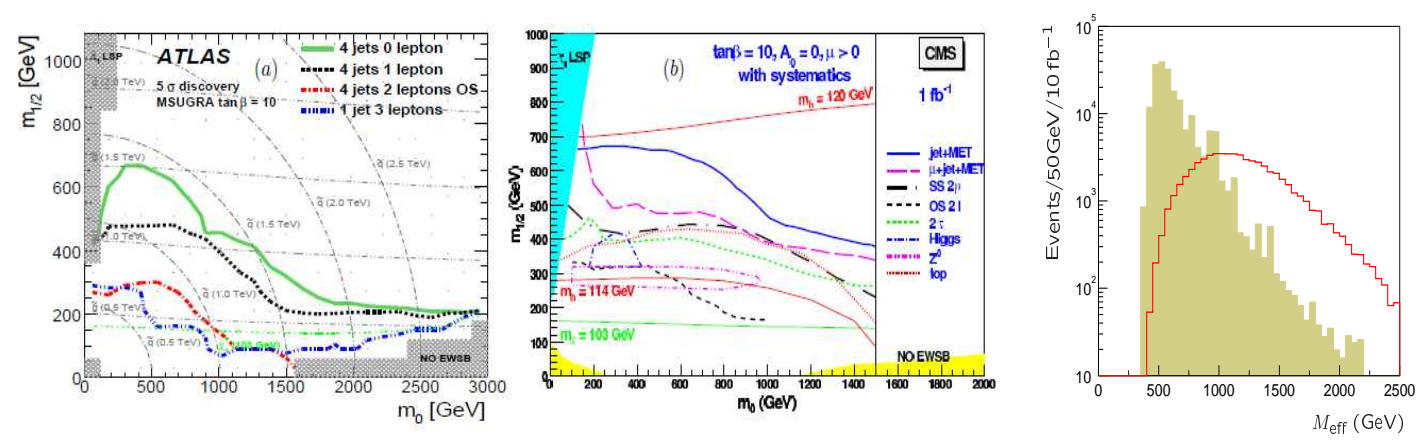

Figure 3: ATLAS and CMS search limits for various channels in the mSUGRA parameter space (left and middle). $M_{\mathrm{eff}}$ distribution for a mSUGRA point and SM background after cuts.

When the sparticle masses become degenerate a reduced probability of events with high $p_{t}$ jets is then expected as well as lower $M_{\text {eff }}$ and $\not_{t}$ making them less "transverse". This means that standard SUSY cuts reduce the signal sample and SUSY discovery is more affected by the SM 
background. If e.g. $m_{\tilde{\chi}_{1}^{0}}>m_{\tilde{q}, \tilde{g}} / 2$, the signal $M_{\text {eff }}$ distribution becomes quite similar to that of the background. However, it has been found [27] that the SUSY signal in the degenerate case exhibits a special universal pattern in $M_{\text {eff }}$ and $E_{t}$ plane which may help to identify the signal region and discriminate signal from background better.

While other $R$-parity conserving models of SUSY breaking are quite different, like the anomalymediation, the reach in $m_{\tilde{q}}, m_{\tilde{g}}$ is similar $\sim 2 \mathrm{TeV}$. It follows from the fact that the overall reach depends mainly on the production cross section as long as there are sufficiently large mass gaps between sparticle masses.

\subsubsection{Sparticle mass measurements}

If $R$-parity is conserved, all SUSY particles decay into invisible LSP, so no mass peaks can be identified. Nevertheless, it might be possible to identify particular decay chains and exploit the "endpoint method" to measure combinations of masses [28]. For example, a relatively clean channel is provided by the three-body decay or, if the slepton can be on-shell, the cascade of twobody decays of the heavier neutralino

$$
\tilde{\chi}_{i}^{0} \rightarrow(\tilde{\ell} \ell) \rightarrow \ell \ell \tilde{\chi}_{1}^{0}
$$

The di-lepton mass distribution endpoints depend on the sparticle masses

$$
\begin{aligned}
& m_{\ell \ell}(\text { 3-body })=m_{\tilde{\chi}_{i}^{0}}-m_{\tilde{\chi}_{1}^{0}} \\
& m_{\ell \ell}(2 \text {-body })=\sqrt{\left(m_{\tilde{\chi}_{i}^{0}}^{2}-m_{\tilde{\ell}}^{2}\right)\left(m_{\tilde{\ell}}^{2}-m_{\tilde{\chi}_{1}^{0}}\right)} / m_{\tilde{\ell}}
\end{aligned}
$$

The events can be searched for by requiring two isolated leptons in addition to multi-jet and $E_{t}$ cuts like those described above. If lepton flavors are separately conserved, then contributions from two uncorrelated decays cancel in the combination of $e^{+} e^{-}+\mu^{+} \mu^{-}-e^{ \pm} \mu^{\mp}$ giving a very clean signal and allowing a precise endpoint measurement. The shape of the distribution also allows us to distinguish two-body from three-body decays.

Long decay chains allow more endpoint measurements. For example, in the SPS1a mSUGRA scenario the following decay chain

$$
\tilde{g} \rightarrow j_{1} \tilde{q} \rightarrow \tilde{\chi}_{2}^{0} j_{1} j_{2} \rightarrow \tilde{\ell} \ell_{1} j_{1} j_{2} \rightarrow \tilde{\chi}_{1}^{0} \ell_{1} \ell_{2} j_{1} j_{2}
$$

can be exploited. With two jets and two leptons in the final state it should be possible to measure the endpoints of invariant mass distributions $\ell \ell, \ell \ell j, \ell j$. These endpoints are smeared by jet reconstruction, hadronic resolution, and miss-assignment of the jets that come from squark decays. Nevertheless, it has been shown [29] that for the integrated luminosity of $300 \mathrm{fb}^{-1}$ these endpoints should be measured at the level of $1 \%$, i.e. determining mass relations to $1-2 \%$. In fact, with so many endpoints one can solve for the absolute values of the unknown masses of $\tilde{g}, \tilde{q}, \tilde{\chi}{ }_{2}^{0}, \tilde{\ell}$ and $\tilde{\chi}_{1}^{0}$ within 5-10\% accuracy. This is a general feature of the determination of sparticle masses when the LSP momentum cannot be measured directly. Nevertheless, $\mathcal{O}(5) \%$ accuracy in the mass of sleptons and the lightest neutralino provides a link to cosmology. With this information one can calculate the neutralino annihilation rate at the time of decoupling and estimate the amount of DM at the level of $7 \%$ [30]. 
It is notable that the LHC can access the mass of the heaviest neutralino $\tilde{\chi}_{4}^{0}$ which in this model is too heavy to be produced at the $500 \mathrm{GeV} e^{+} e^{-}$collider. The measured mass difference $m_{\tilde{\chi}_{4}^{0}}-m_{\tilde{\chi}_{1}^{0}}$, in the same decay chain as in eq.(4.5), but with $\tilde{\chi}_{4}^{0}$ replacing $\tilde{\chi}_{2}^{0}$, directly constrains the $\mu$ parameter. The errors for the MSSM Lagrangian parameters would significantly be reduced if the measurements at the LHC and ILC could be combined [31]. The LHC/ILC interplay is even more important in scenarios with heavy sparticles, like in the cosmology-motivated focus-point scenario [32] in which only limited amount of complementary information from each collider alone can be exploited [33].

If the LSP mass could be measured at the ILC, then errors on the sparticle masses would be reduced significantly, to $\sim 1 \%$ for squark and gluino masses (dominated by the $1 \%$ jet scaling error), and well below $1 \%$ level for weakly interacting sparticles in the SPS1a scenario [34]. In such a case the collider-based calculations of the DM could match the expected accuracy of the Planck probe [35] providing a strong consistency test of particle physics and cosmology.

The mass determination through the endpoint method has several shortcomings: the LSP momentum cannot be reconstructed except for a few very special points in the parameter space, only events near endpoints are used neglecting independent information contained in events away, and the selected events may contain contributions from several cascade decays causing additional systematic uncertainties. These problems can be ameliorated by using the "mass relation" method [36]. In this method the on-shell conditions for sparticle masses in the decay chain are used to solve for the kinematics and reconstruct the SUSY masses as peaks in certain distributions. For example, in the cascade decay eq.(4.5) five on-shell conditions can be written for $\tilde{g}, \tilde{q}, \tilde{\chi}_{2}^{0}, \tilde{\ell}$ and $\tilde{\chi}_{1}^{0}$ in terms of the measured momenta of leptons, jets and 4 unknown momentum components of the undetected neutralino. Each event, therefore, spans a 4-dim hypersurface in a 5-dim mass space, and in principle 5 events would be enough to solve for masses of involved sparticles. Note that events need not be close to endpoints of the decay distributions, i.e. the method can be used even if the number of signal events is small.

\subsubsection{Is it SUSY?}

After careful calibration of LHC detectors and years of collecting data and determining masses of new particles, can we be sure that we see sparticles? Establishing SUSY at the LHC will require not only to discover new particles, measure their masses, decay branching ratios, production cross sections, but also to verify that they are superpartners, i.e. measure their spins and parities, gauge quantum numbers and couplings. A generic weak-scale SUSY signal of large $E_{t}$ arises in almost any model with the lightest $\mathcal{O}(100 \mathrm{GeV})$ particle stable and neutral, as suggested by the dark matter of the universe. Therefore, we should be able to distinguish the SUSY decay chain eq.(4.5) from, e.g., the cascade decay

$$
g^{\prime} \rightarrow j_{1} q^{\prime} \rightarrow Z^{\prime} j_{1} j_{2} \rightarrow \ell^{\prime} \ell_{1} j_{1} j_{2} \rightarrow \gamma^{\prime} \ell_{1} \ell_{2} j_{1} j_{2}
$$

that arises in the universal extra-dimension model (UED) [37]. Here the primes denote the first excited Kaluza-Klein states of the corresponding SM particles with the mass spectrum similar to the SUSY case. In both cases the final state is the same $\ell_{1} \ell_{2} j_{1} j_{2}$ with either the $\tilde{\chi}_{1}^{0}$ or the $\gamma^{\prime}$ escaping detection. What differentiates the decays in eqs. $(4.5,4.6)$ is the spins of intermediate states and the 
chiral structure of couplings. Note that in contrast to the UED case, the SUSY particles are naturally polarized in many processes. For example, in the sub-chain $\tilde{q}_{L} \rightarrow \tilde{\chi}_{2}^{0} q_{L} \rightarrow \tilde{\ell}_{R} \ell_{R} q \rightarrow \tilde{\chi}_{1}^{0} \ell \ell q$ the $\tilde{\chi}_{2}^{0}$ is polarized as right-handed, opposite to $q_{L}$, because the $\tilde{q} \tilde{\chi} q$ Yukawa coupling flips chirality. The polarized neutralino further decays into either $\tilde{\ell}_{R} \ell^{+}$or $\tilde{\ell}_{R}^{*} \ell^{-}$with equal rates (because of the Majorana character of neutralinos). However, due to the chiral nature of the Yukawa $\tilde{\ell} \tilde{\chi} \ell$ coupling, the $\ell^{+}$is likely to fly in the neutralino direction in the squark rest frame, while the $\ell^{-}$in the direction of the quark jet. The difference in the angular distribution is reflected as a charge asymmetry in the invariant mass distribution of the jet-lepton system [38].

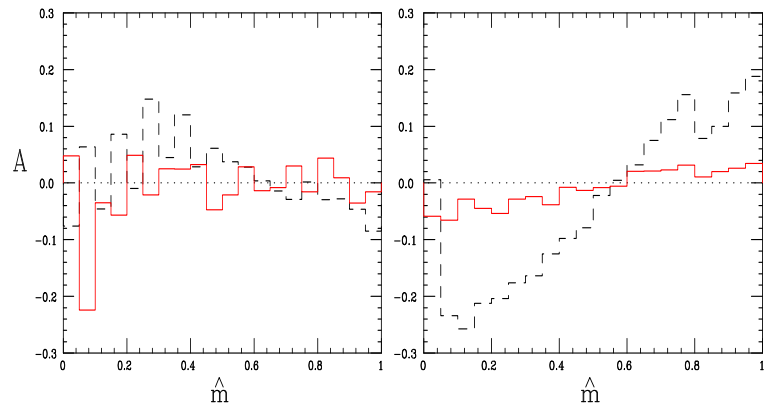

Figure 4: Detector-level charge asymmetries with respect to the jet+lepton rescaled invariant mass, for UED- (left) and SUSY-like (right) mass spectra. Dashed: SUSY. Solid/red: UED.

Although the charge asymmetry for $\tilde{q}_{L}^{*}$ decay is just opposite, in $p p$ collisions more squarks than anti-squarks are expected and the $\tilde{\chi}_{2}^{0}$ production from squark decays is dominant. The amount of charge asymmetry in the $m(j \ell)$ is model dependent, Fig. 4. Nevertheless it remains allowing to resolve the fermionic nature of the neutralino from the vector nature of the $Z^{\prime}$ and confirm the chiral structure of couplings $[39,40]$. Certainly, new ideas to exploit specific features of SUSY at the LHC, for example how to measure the jet charge, are very much welcome.

\subsection{Searches at linear colliders}

If the superpartner masses (at least some of them) are in the TeV range, LHC will certainly see SUSY. Many different channels, in particular from squark and gluino decays will be explored and many interesting quantities measured, as discussed in the previous chapter. However, to prove SUSY one has to scrutinize its characteristic features in as model-independent a way as possible. We will have to:

- measure sparticle masses, their decay widths, production cross sections, mixing angles etc.,

- prove they are superpartners: check their spin, parity, quantum numbers and couplings,

- reconstruct the low-energy SUSY breaking parameters with minimum assumptions,

- and ultimately shed light on physics at the high (GUT?, Planck?) scale.

In answering all the above points an $e^{+} e^{-}$LC would be an indispensable tool [41]. First, the LC will provide independent checks of the LHC findings. Second, thanks to the LC unique features: clean environment, tunable collision energy, high luminosity, polarized incoming beams, and possibly $e^{-} e^{-}$, e $\gamma$ and $\gamma \gamma$ modes, it will offer precise measurements of masses, couplings, quantum numbers, mixing angles, $\mathrm{CP}$ phases etc. Last, but not least, it will provide additional experimental input to the LHC analyses, like the mass of the LSP. Coherent analyses of data from the LHC and LC would thus allow for a better, model independent reconstruction of low-energy SUSY parameters, and connect low-scale phenomenology with the high-scale physics. The interplay between LHC and LC is investigated in detail in the LHC/LC Study Group [42]. 
An intense R\&D process and physics studies since 1992 has lead to world-wide consensus that the next high energy machine after the LHC should be an International Linear Collider (ILC). Planning, designing and funding the ILC requires global participation and global organization. Therefore the Global Design Effort for the ILC [43], headed by Barry Barish, has been established with the goal of producing an ILC Reference Design Report by the beginning of 2007, an ILC Technical Design Report by the end of 2008 and be ready for construction around 2010. The ILC baseline design foresees:

- CM energy adjustable from 200 to $500 \mathrm{GeV}$, and at $M_{Z}$ for calibration,

- integrated luminosity of at least $500 \mathrm{fb}^{-1}$ in first 4 years,

- beam energy stability and precision below $1 \%$,

- electron beam polarization of at least $80 \%$,

- upgradeability to CM energy of $1 \mathrm{TeV}$.

The choice of options, like GigaZ (high luminosity run at $M_{Z}$ ), positron polarization, $e^{-} e^{-}, e \gamma$ or $\gamma \gamma$, will depend on LHC+ILC physics results.

Many detailed physics calculations and simulations have been performed and presented during numerous ECFA, ACFA and ALCPG workshops and LCWS conferences [44]. Below only some highlights are presented. For more examples and references I refer to my reviews [45].

\subsubsection{Mass measurements}

Sparticle masses can be measured in threshold scans or in continuum. For first 2 generations, where $R-L$ mixing can be neglected, the shape of the production cross section near threshold is sensitive to the masses and quantum numbers. For example, for selectrons and smuons, $\tilde{\mu}_{L}^{+} \tilde{\mu}_{L}^{-}, \tilde{\mu}_{R}^{+} \tilde{\mu}_{R}^{-}, \tilde{e}_{L}^{+} \tilde{e}_{L}^{-}$and $\tilde{e}_{R}^{+} \tilde{e}_{R}^{-}$pairs are excited in a P-wave characterized by a slow rise of the cross section $\sigma \sim \beta^{3}$ with slepton velocity $\beta$. On the other hand, in $e_{L}^{+} e_{L}^{-} / e_{R}^{+} e_{R}^{-} \rightarrow \tilde{e}_{R}^{+} \tilde{e}_{L}^{-} / \tilde{e}_{L}^{+} \tilde{e}_{R}^{-}$ and $e_{L}^{-} e_{L}^{-} / e_{R}^{-} e_{R}^{-} \rightarrow \tilde{e}_{L}^{-} \tilde{e}_{L}^{-} / \tilde{e}_{R}^{-} \tilde{e}_{R}^{-}$sleptons are excited in the $S$-wave giving steep rise of the cross sections $\sigma \sim \beta$. The expected experimental precision requires higher order corrections, and finite sfermion width effects to be included. Examples of simulations for the SPS1a point are shown in Fig. 5 [46]. Using polarized $e^{+} e^{-}$beams and $50 \mathrm{fb}^{-1}$ the $\tilde{e}_{R}$ mass can be determined to 2 per mil; the resolution deteriorates by a factor of $\sim 2$ for $\tilde{\mu}_{R}^{+} \tilde{\mu}_{R}^{-}$production. For $e_{R}^{-} e_{R}^{-} \rightarrow \tilde{e}_{R} \tilde{e}_{R}$ the gain in resolution is a factor $\sim 4$ with only a tenth of the luminosity, compared to $e^{+} e^{-}$beams. Above
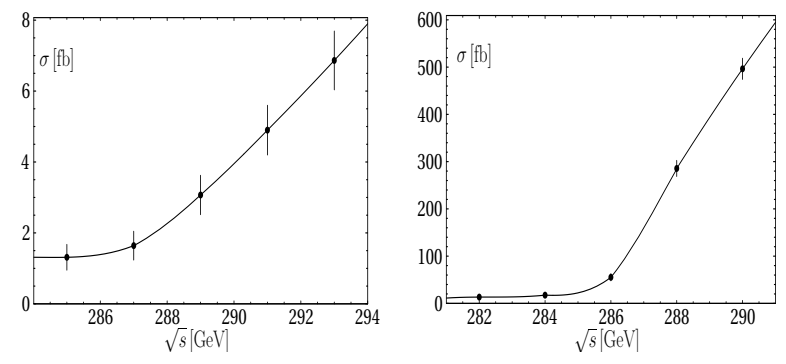

Figure 5: Cross sections at threshold for the reactions $e_{L}^{+} e_{R}^{-} \rightarrow \tilde{e}_{R}^{+} \tilde{e}_{R}^{-}$(left) and $e_{R}^{-} e_{R}^{-} \rightarrow \tilde{e}_{R}^{-} \tilde{e}_{R}^{-}$ (right) in the SPS1a scenario, including background. Error bars correspond to a luminosity of $10 \mathrm{fb}^{-1}$ (left) and $1 \mathrm{bf}^{-1}$ (right) per point.

the threshold, slepton masses can be obtained from the endpoint energies of leptons coming from slepton decays. In the case of two-body decays, $\tilde{\ell}^{-} \rightarrow \ell^{-} \tilde{\chi}_{i}^{0}$ and $\tilde{\nu}_{\ell} \rightarrow \ell^{-} \tilde{\chi}_{i}^{+}$the lepton energy spectrum is flat with endpoints (the minimum $E_{-}$and maximum $E_{+}$energies)

$$
E_{ \pm}=\frac{1}{4} \sqrt{s}(1 \pm \beta)\left(1-m_{\tilde{\chi}}^{2} / m_{\tilde{\ell}}^{2}\right)
$$


providing an accurate determination of the masses of the primary slepton and the secondary neutralino/chargino.

Simulations of the $e$ and $\mu$ energy spectra of $\tilde{e}_{R} \tilde{e}_{R}$ and $\tilde{\mu}_{R} \tilde{\mu}_{R}$ (respectively) production, including beamstrahlung, QED radiation, selection criteria and detector resolutions, are shown in Fig. 6 assuming mSUGRA scenario SPS1a [47]. With a moderate luminosity of $200 \mathrm{fb}^{-1}$ at $\sqrt{s}=400$ $\mathrm{GeV}$ one finds $m_{\tilde{e}_{R}}=143 \pm 0.10 \mathrm{GeV}, m_{\tilde{\mu}_{R}}=143 \pm 0.10 \mathrm{GeV}$ and $m_{\tilde{\chi}_{1}^{0}}=96 \pm 0.10 \mathrm{GeV}$ from selectron, or $m_{\tilde{\chi}_{1}^{0}}=96 \pm 0.18 \mathrm{GeV}$ from smuon production processes. Assuming the neutralino mass is known, one can improve slepton mass determination by a factor 2 from reconstructed kinematically allowed minimum $m_{\min }(\tilde{\ell})$. The partner $\tilde{\mu}_{L}$ is more difficult to detect because of large background from $W W$ pairs and SUSY cascades. However, with the high luminosity of TESLA one may select the rare decay modes $\tilde{\mu}_{L} \rightarrow \mu \tilde{\chi}_{2}^{0}$ and $\tilde{\chi}_{2}^{0} \rightarrow \ell^{+} \ell^{-} \tilde{\chi}_{1}^{0}$, leading to a unique, background free signature $\mu^{+} \mu^{-} 4 \ell^{ \pm} \not E$. The achievable mass resolutions for $m_{\tilde{\mu}_{L}}$ and $m_{\tilde{\chi}_{2}^{0}}$ is of the order of $0.4 \mathrm{GeV}$ [48].
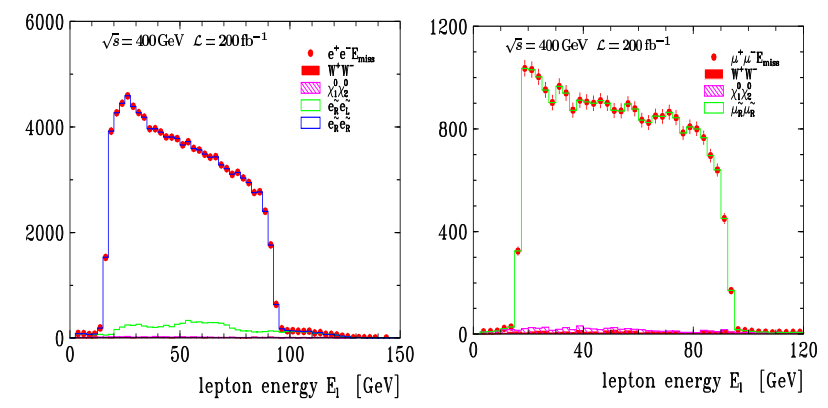

Figure 6: Lepton energy spectra in the processes $e_{R}^{-} e_{L}^{+} \rightarrow \tilde{e}_{R}^{-} \tilde{e}_{R}^{+} \rightarrow$ $e^{-} \tilde{\chi}_{1}^{0} e^{+} \tilde{\chi}_{1}^{0}$ (left) and $e_{R}^{-} e_{L}^{+} \rightarrow \tilde{\mu}_{R}^{-} \tilde{\mu}_{R}^{+} \rightarrow$ $\mu^{-} \tilde{\chi}_{1}^{0} \mu^{+} \tilde{\chi}_{1}^{0} \rightarrow \mu^{-} \tilde{\chi}_{1}^{0} \mu^{+} \tilde{\chi}_{1}^{0}$ (right) at $\sqrt{s}=400 \mathrm{GeV}$ and luminosity $200 \mathrm{fb}-1$; scenario SPS1a.

In a similar fashion the chargino masses can be measured very precisely at threshold: simulations for the reaction $e_{R}^{+} e_{L}^{-} \rightarrow \tilde{\chi}_{1}^{+} \tilde{\chi}_{1}^{-} \rightarrow \ell^{ \pm} \nu_{\ell} \tilde{\chi}_{1}^{0} q \bar{q}^{\prime} \tilde{\chi}_{1}^{0}$ show that the mass resolution is excellent of $\mathcal{O}(50 \mathrm{MeV})$, degrading to the per mil level for the higher $\tilde{\chi}_{2}^{ \pm}$state. Above threshold, from the di-jet energy distribution one expects a mass resolution of $\delta m_{\tilde{\chi}_{1}^{ \pm}}=0.2 \mathrm{GeV}$, while the di-jet mass distributions constrains the $\tilde{\chi}_{1}^{ \pm}-\tilde{\chi}_{1}^{0}$ mass splitting within about $100 \mathrm{MeV}$. Similarly to the chargino case, the di-lepton energy and mass distributions in the reaction $e^{+} e^{-} \rightarrow \tilde{\chi}_{2}^{0} \tilde{\chi}_{2}^{0} \rightarrow 4 \ell^{ \pm} \mathbb{E}$ can be used to determine $\tilde{\chi}_{1}^{0}$ and $\tilde{\chi}_{2}^{0}$ masses. Previous analyses of the di-lepton mass and di-lepton energy spectra performed in the $\tan \beta=3$ case showed that uncertainties in the primary and secondary $\tilde{\chi}_{2}^{0}$ and $\tilde{\chi}_{1}^{0}$ masses of about 2 per mil can be expected [41, 48]. Higher resolution of order $100 \mathrm{MeV}$ for $m_{\tilde{\chi}_{2}^{0}}$ can be obtained from a threshold scan of $e^{+} e^{-} \rightarrow \tilde{\chi}_{2}^{0} \tilde{\chi}_{2}^{0}$; heavier states $\tilde{\chi}_{3}^{0}$ and $\tilde{\chi}_{4}^{0}$, if accessible, can still be resolved with a resolution of a few hundred $\mathrm{MeV}$.

\subsubsection{Measuring couplings and mixings}

In contrast to the first two generations, the $L-R$ mixing for the third generation can be nonnegligible due to the large Yukawa coupling. Therefore the $\tilde{\tau}, \tilde{t}$ and $\tilde{b}$ are very interesting to study to determine their mixing and chiral quantum numbers. Similarly, it is very important to measure the gaugino and higgsino composition of charginos and neutralinos. Last, but not least, we have to verify SUSY mass relations and exact equality (at tree level) of gauge couplings and their supersymmetric Yukawa counterparts.

In this respect the ability of having both beams, positrons and electrons, polarised is particularly important [49], since for many measurements even $100 \%$ electron polarisation is insufficient. 
The couplings and mixing angles can be extracted from production cross sections measured with polarized beams. For example, experimental analyses of stop quarks with small stop-neutralino mass difference have been performed [50]. Such analyses are motivated by the stop-neutralino coannihilation scenario consistent with dark matter relic density and EW baryogenesis. With small stop-neutralino mass difference, the stop decays into neutralino and charm making the analysis very demanding. Nevertheless, the stop parameters can be determined precise enough, Fig. 7, and precisions for the dark matter predictions comparable to that from direct WMAP measurements in the region down to mass differences $\sim \mathcal{O}(5 \mathrm{GeV})$ can be achieved [50].

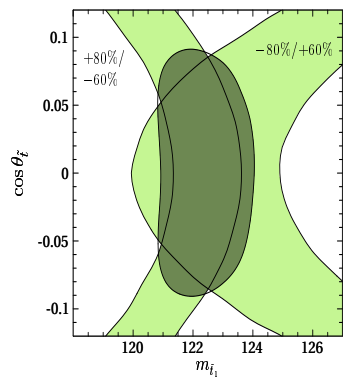

(a)

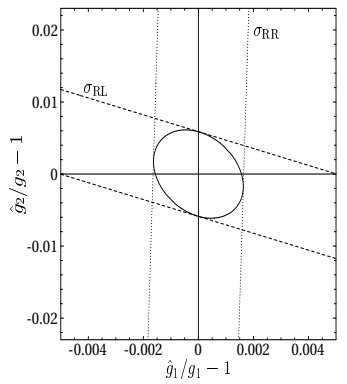

(b)

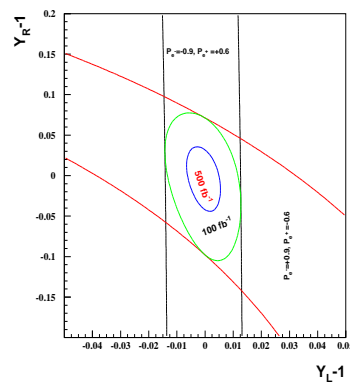

(c)

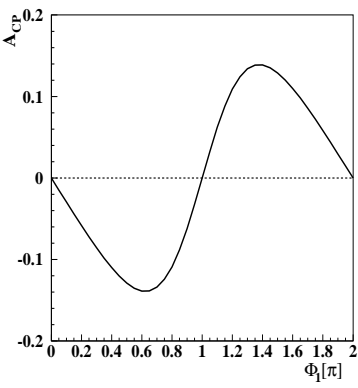

(d)

Figure 7: Power of polarization - bounds on: (a) light stop mass $m_{\tilde{t}_{1}}$ and stop mixing angle $\theta_{\tilde{t}}$ from $\sigma\left(e^{+} e^{-} \rightarrow \tilde{t}_{1} \tilde{t}_{1}^{*}\right) ;(b)$ on supersymmetric Yukawa couplings $\hat{g}_{1}$ and $\hat{g}_{2}$ from selectron cross-section measurements; (c) on $Y_{L}=\hat{g}_{2} / g_{2}$ and $Y_{R}=\hat{g}_{1} / g_{1}$ from neutralino pair-production with polarized beams. (d) $\Phi_{1}$ dependence of the $C P-$ odd asymmetry $A_{\mathrm{CP}}$.

The Yukawa couplings of scalar fermions can be precisely determined by measuring the production cross-sections with polarized beams. For example, in the electroweak sector, the relation between the hypercharge $\mathrm{U}(1)_{\mathrm{Y}}$ coupling $g_{1}$ and the $\mathrm{SU}(2)_{\mathrm{L}}$ coupling $g_{2}$ and the corresponding Yukawa couplings $\hat{g}_{1}$ and $\hat{g}_{2}$ can accurately be tested by measuring the pair-production crosssections of scalar leptons. The resulting constraints on from selectron cross-section measurements are depicted in Fig. 7(b), from [51]. The identity of the SUSY Yukawa and gauge couplings can also be independently checked in neutralino pair-production. Combining the measurements of $\sigma_{R}$ and $\sigma_{L}$ for the process $e^{+} e^{-} \rightarrow \tilde{\chi}_{1}^{0} \tilde{\chi}_{2}^{0}$, the Yukawa couplings can be determined to quite a high precision, as demonstrated in Fig. 7(c) [52].

Polarisation is a very powerful tool not only for preparing the desirable initial state, but also as a diagnosis tool of final states. For example, neutralinos $\tilde{\chi}_{2}^{0}$ produced in $\tilde{e}_{L}^{ \pm}$decays are $100 \%$ polarized [53]. Furthermore, in $e^{+} e^{-} \rightarrow \tilde{e}_{L}^{+} \tilde{e}_{L}^{-} \rightarrow e^{+} \tilde{\chi}_{1}^{0} e^{-} \tilde{\chi}_{2}^{0}$ followed by the three-body decay $\tilde{\chi}_{2}^{0} \rightarrow \tilde{\chi}_{1}^{0} \mu^{+} \mu^{-}$it is possible to reconstruct the rest frame of the neutralino $\tilde{\chi}_{2}^{0}$ as shown in Ref. [54]. Such a perfect neutralino polarization combined with the study of angular correlations in the neutralino rest frame can provide us with ways for probing the Majorana nature of the neutralinos and $\mathrm{CP}$ violation in the neutralino system. With the neutralino spin vector $\hat{n}$ and two final lepton momentum directions $\hat{q}^{+}$and $\hat{q}^{-}$the CP-odd asymmetry can be constructed by comparing number of events with $O_{\mathrm{CP}}=\hat{n} \cdot\left(\hat{q}_{+} \times \hat{q}_{-}\right)$positive and negative, normalized to the sum. Fig. 7(d) shows the dependence of the CP-odd asymmetry on the phase $\Phi_{1}$ of the Bino mass parameter $M_{1}$ [55]. 


\subsubsection{Beyond the kinematic reach}

The precision measurements offered by the ILC allow us to infer indirect information on heavy states not directly accessible at the first stage ILC. I will present two examples.

If the heavier stop $\tilde{t}_{2}$ is too heavy for the ILC, and due to huge background invisible at the LHC, the precise measurement of the Higgs boson mass $m_{h}$ together with measurements from the LHC can be used to obtain indirect limits on $m_{\tilde{t}_{2}}$ [56]. Intersection of the measured value $m_{h}=$ $115.5 \pm 0.05 \mathrm{GeV}$ with the allowed $m_{\tilde{t}_{2}}-m_{h}$ region, Fig. 8 (left), gives an indirect determination of $m_{\tilde{t}_{2}}$.

There are interesting scenarios in which scalar sparticle sector is heavy while the gaugino masses are kept relatively small, like for instance in focus-point scenarios. Precision analyses of cross sections for light chargino production and forward-backward asymmetries of decay leptons at the first stage of the ILC, Fig. 8 (right), together with mass information on $\tilde{\chi}_{2}^{0}$ and squarks from the LHC, show that the underlying fundamental gaugino/higgsino MSSM parameters and constrains on the heavy, kinematically inaccessible sparticles with masses $\mathcal{O}(2 \mathrm{TeV})$, can be obtained nevertheless [33].
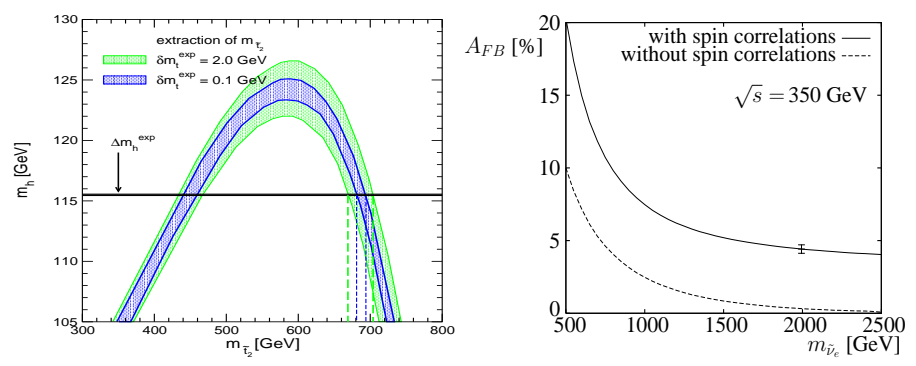

Figure 8: Indirect determination of: $m_{\tilde{t}_{2}}$ from the $m_{h}$ measurement (left); $m_{\tilde{\nu}_{e}}$ from the forward-backward asymmetry of $e^{-}$in the process $e^{+} e^{-} \rightarrow \tilde{\chi}_{1}^{+} \tilde{\chi}_{1}^{-}, \tilde{\chi}_{1}^{-} \rightarrow \tilde{\chi}_{1}^{0} \ell^{-} \bar{\nu}($ right $)$.

The above examples stress again the important role of the LHC/ILC interplay, since neither of these colliders alone can provide us with the data needed to determine the SUSY parameters in such scenarios. Also the derived constraints on heavy, kinematically inaccessible sparticles may provide the physics argument for a second stage of the ILC.

\subsection{4 $e^{-} e^{-}, e \gamma$ and $\gamma \gamma$ options}

Compton back-scattering of the laser light on the electron beam(s) opens a possibility of converting the $e^{-} e^{-}$collider to an $e \gamma$ and $\gamma \gamma$ collider with energies and luminosities comparable to those of $e^{+} e^{-}$collider [57]. If realized, these options may open new discovery channels. Again I will take two specific examples to illustrate the point.

If the mass difference between the lightest neutralino and the selectron is a few hundred $\mathrm{GeV}$, it may happen that chargino pair production at the ILC is possible, while selectron pair production is kinematically forbidden. However, $m_{\tilde{\chi}_{1}^{0}}+m_{\tilde{e}}$ can still be below $90 \%$ of the centre-of-mass energy, so that the process $e \gamma \rightarrow \tilde{\chi}_{1}^{0} \tilde{e}^{-}$is possible at an $e \gamma$ collider. If the photon energy were known, the selectron and neutralino masses could be determined from the endpoints of the decay electron distribution, like in $e^{+} e^{-}$collisions. Although the variable photon energy smears the endpoints, simulations have shown (Fig. 9) that with the $m_{\tilde{\chi}_{1}^{0}}$ determined in $e+e^{-}$running, the selectron mass can be reconstructed from the position of the lower edge within $0.3 \%$ [58]. 
$\gamma \gamma$ collider offers a unique possibility of producing as $s$-channel resonances neutral Higgs bosons $H, A$ that are both too heavy to be produced in associated $H A$ or $Z H$ processes at $e^{+} e^{-}$ collider and lay in the so called "LHC-wedge" of intermediate values of $\tan \beta$, to which LHC is blind. Results of a simulation for the combined $\gamma \gamma \rightarrow H, A \rightarrow b \bar{b}$ analyses are shown in Fig. 9, since in this region the $H$ and $A$ bosons are almost mass-degenerate [59]. Other decay modes ( $W W, Z Z, t \bar{t}$ ) can provide a means to determine the Higgs-boson CP properties [60], and the $\tau$ fusion process, $\gamma \gamma \rightarrow \tau \tau H, A$, can serve to measure $\tan \beta$ [61], the parameter that is notoriously difficult to determine experimentally.
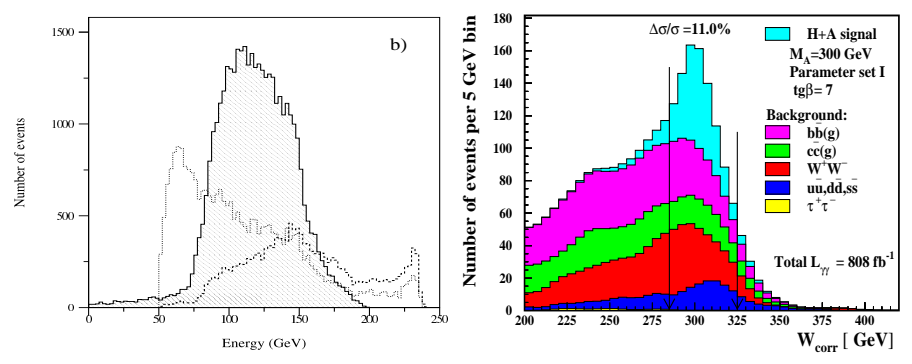

Figure 9: Electron energy distribution signal e $\rightarrow \nu_{e} \nu_{e} e$ (shaded) and background after cuts (left). Invariant mass distribution for $\gamma \gamma$ $H, A \rightarrow b \bar{b}$ and and background (right).

\subsection{Beyond the ILC}

It is expected that higher energy colliders will be needed to help unravel the multi-TeV physics left unveiled either by the LHC or by the ILC. Further progress in particle physics may require clean experiments at a linear $e^{+} e^{-}$collider at multi-TeV energies, like CLIC [62]. With tunable energy and beam polarisation to disentangle various channels, and high luminosity for precision measurements, CLIC would be the ideal machine to complement the the LHC and ILC physics program. Simulations for CLIC concentrated on such scenarios with sparticles beyond the LHC and ILC reach.

Fig. 10 (left) shows simulations of the muon energy spectrum from a $1150 \mathrm{GeV}$ selectron decaying to a muon and a $660 \mathrm{GeV}$ LSP neutralino. The endpoints are clearly seen allowing a 2-3\% accuracy on the selectron and neutralino mass determination. Likewise, in Fig. 10(middle) the di-muon invariant mass distribution from $\tilde{\chi}_{2}^{0} \rightarrow \mu^{+} \mu^{-} \tilde{\chi}_{1}^{0}$ exhibits a pronounced edge which, together with results from selectron decay make a measurement of $m_{\tilde{\chi}_{2}^{0}}$ up to $2 \%$ accuracy possible.
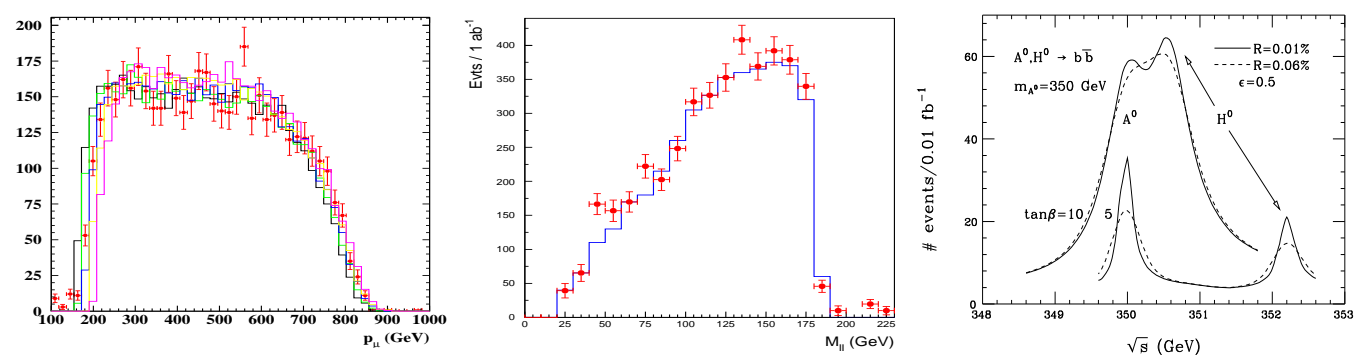

Figure 10: Muon energy spectrum from $\tilde{\mu}_{L} \rightarrow \mu \tilde{\chi}_{1}^{0}$ (left), and di-muon invariant mass spectrum from $\tilde{\chi}_{2}^{0} \rightarrow \mu^{+} \mu^{-} \tilde{\chi}_{1}^{0}$ (middle) at CLIC. Separation of $A$ and H signals at a muon collider (right).

In more distant future a muon collider with extremely good beam energy resolution and enhanced couplings of muons to Higgs bosons will provide a tool to explore Higgs (and Higgs-like 
objects) by direct $s$-channel fusion, much like LEP explored the $Z$. Right panel of Fig. 10 demonstrates how well two almost mass-degenerate Higgs bosons $H$ and $A$ can be resolved [63].

\section{Reconstructing the underlying SUSY model}

The LHC experiments in the supersymmetric particle sector offer not only the discovery potential but also many high precision measurements of masses and couplings. The next step towards establishing SUSY is the reconstruction of low-energy SUSY breaking Lagrangian parameters without assuming a specific scenario. This is a highly non-trivial task [64] stressed recently in [65]. This task will be greatly ameliorated by experimenting at the ILC where the experimental accuracies at the per-cent down to the per-mill level are expected [41]. The ultimate goal of all experimental efforts will be to unravel the SUSY breaking mechanism sheding light on physics at high (GUT?, Planck?) scale.

The expected high experimental accuracies at the LHC/ILC should be matched from the theoretical side. This calls for a well defined theoretical framework for the calculational schemes in perturbation theory as well as for the input parameters. Motivated by the experience in analyzing data at the former $e^{+} e^{-}$colliders LEP and SLC, the SPA Convention and Project [34] has been proposed. It provides: a convention for high-precision theoretical calculations, a program repository of numerical codes, a list of tasks needed further improvements and a SUSY reference point SPS1 $\mathrm{a}^{\prime}$ as a test-bed.

The SPA Convention and Project is a joint inter-regional effort that could serve as a forum to discuss future improvements on both experimental and theoretical sides to exploit fully the physics potential of LHC, and ILC. The current status of the project is documented on the routinely updated web-page http://spa.desy.de/spa/

\subsection{SPA Convention}

Building on vast experience in SUSY calculations and data simulations and analyses, the SPA Convention consists of the following propositions:

- The masses of the SUSY particles and Higgs bosons are defined as pole masses.

- All SUSY Lagrangian parameters, mass parameters and couplings, including $\tan \beta$, are given in the $\overline{D R}$ scheme at the scale $\tilde{M}=1 \mathrm{TeV}$.

- Gaugino/higgsino and scalar mass matrices, rotation matrices and the corresponding angles are defined in the $\overline{D R}$ scheme at $\tilde{M}$, except for the Higgs system in which the mixing matrix is defined in the on-shell scheme, the scale parameter chosen as the light Higgs mass.

- The Standard Model input parameters of the gauge sector are chosen as $G_{F}, \alpha, M_{Z}$ and $\alpha_{s}^{\overline{M S}}\left(M_{Z}\right)$. All lepton masses are defined on-shell. The $t$ quark mass is defined on-shell; the $b, c$ quark masses are introduced in $\overline{M S}$ at the scale of the masses themselves while taken at a renormalization scale of $2 \mathrm{GeV}$ for the light $u, d, s$ quarks.

- Decay widths, branching ratios and production cross sections are calculated for the set of parameters specified above. 


\subsection{Program repository}

The repository contains links to codes grouped in several categories: scheme translation tools for definitions and relations between on-shell, $\overline{D R}$ and $\overline{M S}$ parameters; spectrum calculators from the Lagrangian parameters; calculators of various observables: decay tables, cross sections, lowenergy observables, cold dark matter relics, cross sections for CDM particle searches; event generators; analysis programs to extract the Lagrangian parameters from experimental data; RGE codes; as well as some auxiliary programs and libraries.

The responsibility for developing codes and maintaining them up to the current theoretical state-of-the-art precision rests with the authors. The SLHA [66] convention is recommended for communication between the codes.

\subsection{The test-bed: Ref. Point SPS1a'}

The SPA Convention and Project is set up to cover general SUSY scenarios. However, to perform first checks of its internal consistency and to explore the potential of such coherent data analyses a MSSM Reference Point SPS1a' has been proposed as a testing ground. Of course, in future the SPA has to be tested in more complicated scenarios.

The roots defining the Point SPS1a' are the mSUGRA parameters $M_{1 / 2}=250 \mathrm{GeV}, M_{0}=70$ $\mathrm{GeV}, A_{0}=-300 \mathrm{GeV}$ defined at the GUT scale, and $\tan \beta(\tilde{M})=10, \mu>0$. The point is close to the original Snowmass point SPS1a [19] and to point $B^{\prime}$ of [67].

If SPS1a', or a SUSY scenario with mass scales similar to this point, is realized in nature, a plethora of interesting channels can be exploited to extract the basic supersymmetry parameters when combining experimental information from mass distributions at LHC with measurements of decay spectra and threshold excitation curves at an $e^{+} e^{-}$collider with energy up to $1 \mathrm{TeV}$. Recently global analysis programs have become available [68] in which the whole set of data, masses, cross sections, branching ratios etc., is exploited coherently to extract the Lagrangian parameters in the optimal way after including the available radiative corrections.

\subsection{Future developments}

Although current SPA studies are very encouraging, much additional work both on the theoretical as well as on the experimental side will be needed to achieve the SPA goals. In particular - The present level of theoretical calculations still does not match the expected experimental precision, particularly in coherent LHC+ILC analyses. - There is no complete proof that $\overline{D R}$ scheme preserves supersymmetry and gauge invariance in all cases.

- A limited set of observables included in experimental analyses by no means exhausts the opportunities which data at LHC and at ILC are expected to provide. Most experimental analyses not include the theoretical errors which must be improved considerably before matching the experimental standards.

- The parameter set SPS 1 $\mathrm{a}^{\prime}$ chosen for a first study provides a benchmark for developing and testing the tools needed for a successful analysis of future SUSY data. However, neither this specific point nor the MSSM itself may be the correct model for low-scale SUSY. While versions of mSUGRA and of gaugino mediation have also been analyzed in some detail, the analyses have to be extended systematically to other possibilities. In particular, CP violation, R-parity violation, flavor violation, 
NMSSM and extended gauge groups are among scenarios which might be realized in the SUSY sector. The SPA conventions are general enough to cover all these scenarios.

\subsection{Cosmology connection}

Among the many possible models of dark matter, interesting are models in which the dark matter particle is a massive neutral weakly interacting particle (WIMP) with a mass of the order of $100 \mathrm{GeV}$. In supersymmetry the LSP, neutralino in many SUSY incarnations, can serve as a perfect candidate for WIMP. If so, these particles should be produced in reactions at the next generation of high-energy accelerators. Astrophysical data, therefore, play an increasingly important role in confronting supersymmetry with experiments. On the one hand the relic DM abundance imposes crucial limits on supersymmetric scenarios, on the other, the comprehensive parameter analysis of high-energy experiments should provide insight into the nature of the cold dark matter particles.

Since, by definition, not directly visible to high-energy physics experiments, determination of their properties is necessarily indirect. Moreover, the WIMP cross sections have a complicated dependence on the underlying spectrum parameters, and many of those parameters cannot realistically be measured in high-energy physics experiments. Hopefully, the large number of specific and precision measurements at the LHC and ILC will allow us to determine the underlying particle physics model well enough to predict the microscopic properties of the dark matter. From this, we can predict the cosmic density, the annihilation cross sections, and the cross sections relevant to direct detection.

Last year at Snowmass a set of four benchmarks in mSUGRA has been proposed for detailed simulations [69]. Point LCC1 is identical to SPS1a, in which the dominant channel is the neutralino annihilation to lepton pairs. Point LCC2 has substantial gaugino-higgsino mixing making the annihilation processes to $W^{+} W^{-}, Z^{0} Z^{0}$ and $Z^{0} h^{0}$ dominant. Point LCC3 is characterized by small neutralino-stau mass difference and therefore the coannihilation with $\tilde{\tau}$ is important. Finally, for point LCC4 the $A^{0}$ resonance makes an important contribution to the neutralino annihilation cross section.

In some scenarios (for example LCC1) the measurements at the LHC combined with the assumption that supersymmetry is the correct model of new physics provides quite accurate prediction of the WIMP relic density. In most scenarios, however, the LHC data alone will not be sufficient even if SUSY is assumed to be the underlying theory. Fortunately, the estimates of the neutralino properties would be dramatically improved when the neutralino and the other light sparticles are observed in $e^{+} e^{-}$annihilation at the $\operatorname{ILC}[34,35,70]$.

\section{Summary}

Much progress has been achieved during last years in constraining SUSY at existing colliders and preparing the physics programme for new machines. At the beginning the LHC has been considered merely as a discovery machine. However, over the years many techniques have been developed for extracting masses and couplings, and in some cases the Lagrangian parameters. On the theory side many higher-order calculations have been completed and implemented in numerical codes. New theoretical ideas are popping up that deserve experimental analyses. To complete the task of exploring all masses and couplings of SUSY particles is probably impossible by the LHC 
alone. The ILC will extend the discovery reach, in particular in the electroweak sector, and greatly improve on precision SUSY measurements. We still need new ideas and techniques to explore fully the opportunities offered to us by the LHC and ILC, and in a distant future at CLIC. But most of all, we need data, and first hints of SUSY at the LHC.

\section{Acknowledgments}

I would like to thank the organizers of the school for creative atmosphere, warm hospitality and support. I am also grateful to the Theory Division for the hospitality at CERN where this manuscript has been completed. Partial support by the Polish Ministry of Science and Higher Education Grant N N202 230337 and the EC Programme"Particle Physics and Cosmology: the Interface" (MTKD-CT-2005-029466) is greatly acknowledged.

\section{References}

[1] P. Fayet and S. Ferrara, "Supersymmetry,” Phys. Rept. 32 (1977) 249.

H. P. Nilles, "Supersymmetry, Supergravity And Particle Physics," Phys. Rept. 110, 1 (1984).

H. E. Haber and G. L. Kane, "The Search For Supersymmetry: Probing Physics Beyond The Standard Model,” Phys. Rept. 117 (1985) 75.

J. Wess and J. Bagger, "Supersymmetry and supergravity," Princeton, USA: Univ. Pr. (1992)

M. Drees, “An introduction to supersymmetry,” arXiv:hep-ph/9611409.

J. D. Lykken, arXiv:hep-th/9612114.

S. P. Martin, “A Supersymmetry Primer,” arXiv:hep-ph/9709356.

K. A. Olive, "Introduction to supersymmetry: Astrophysical and phenomenological arXiv:hep-ph/9911307.

M. Drees, R. Godbole and P. Roy, "Theory and phenomenology of sparticles: An account of four-dimensional N=1 supersymmetry in high energy physics," Hackensack, USA: World Scientific (2004)

K. A. Intriligator and N. Seiberg, "Lectures on Supersymmetry Breaking," Class. Quant. Grav. 24 (2007) S741 [arXiv:hep-ph/0702069].

M. E. Peskin, “Supersymmetry in Elementary Particle Physics,” arXiv:0801.1928 [hep-ph].

H. K. Dreiner, H. E. Haber and S. P. Martin, "Two-component spinor techniques and Feynman rules for quantum field theory and supersymmetry," arXiv:0812.1594 [hep-ph].

M. Dine, "Supersymmetry Breaking at Low Energies," arXiv:0901.1713 [hep-ph].

[2] ALEPH, DELPHI, L3, OPAL and SLD Collaborations and LEPEW, SLDEW and SLD Heavy Flavour Working Groups, Precision electroweak measurements on the Z resonance, Phys. Rept. 427 (2006) 257.

[3] Yu. A. Golfand and E. P. Likhtman, JETP Lett. 13 (1971) 323 [Pisma Zh. Eksp. Teor. Fiz. 13 (1971) 452]. D. V. Volkov and V. P. Akulov, JETP Lett. 16 (1972) 438 [Pisma Zh. Eksp. Teor. Fiz. 16 (1972) 621].

[4] J. Wess and B. Zumino, Phys. Lett. B 49 (1974) 52.

[5] A. Signer, J. Phys. G 36 (2009) 073002 [arXiv:0905.4630 [hep-ph]].

[6] S. R. Coleman and J. Mandula, Phys. Rev. 159 (1967) 1251. 
[7] In 1968 H. Miyazawa considered composite spinor operators that transformed baryons to mesons, see H. Miyazawa, Phys. Rev. 170 (1968) 1586.

[8] R. Haag, J. T. Lopuszanski and M. Sohnius, Nucl. Phys. B 88, 257 (1975).

[9] M. T. Grisaru, W. Siegel and M. Rocek, Nucl. Phys. B 159, 429 (1979).

[10] Y. Shirman, arXiv:0907.0039 [hep-ph].

[11] N. Seiberg, Phys. Lett. B 318 (1993) 469 [arXiv:hep-ph/9309335].

[12] P. Fayet and J. Iliopoulos, Phys. Lett. B 51 (1974) 461.

[13] P. Fayet, Phys. Lett. B 69 (1977) 489. G. R. Farrar and P. Fayet, Phys. Lett. B 76 (1978) 575.

[14] L. Girardello and M. T. Grisaru, Nucl. Phys. B 194 (1982) 65.

[15] J. Rosiek, Phys. Rev. D 41 (1990) 3464;

J. Rosiek, arXiv:hep-ph/9511250.

[16] T. Appelquist and J. Carazzone, Phys. Rev. D 11 (1975) 2856.

[17] G. Hinshaw et al. [WMAP Collaboration], Astrophys. J. Suppl. 180 (2009) 225 [arXiv:0803.0732 [astro-ph]].

[18] O. Buchmueller et al., JHEP 0809 (2008) 117 [arXiv:0808.4128 [hep-ph]].

[19] B. C. Allanach et al., Eur. Phys. J. C 25 (2002) 113.

[20] G. A. Blair, W. Porod and P. M. Zerwas, Phys. Rev. D 63 (2001) 017703 and Eur. Phys. J. C 27 (2003) 263. B. C. Allanach, D. Grellscheid and F. Quevedo, JHEP 0205 (2002) 048. G. L. Kane, J. Lykken, S. Mrenna, B. D. Nelson. L. T. Wang and T. T. Wang, Phys. Rev. D 67 (2003) 045008.

[21] http://www-cdf.fnal.gov/physics/exotic/exotic.html http://www-d0.fnal.gov/Run2Physics/WWW/results/np.htm http://www-h1.desy.de/h1/www/publications/H1_sci_results.shtml http://www-zeus.desy.de/publications.php3.

[22] V. M. Abazov et al. [D0 Collaboration], Phys. Lett. B 660 (2008) 449 [arXiv:0712.3805 [hep-ex]]. T. Aaltonen et al. [CDF Collaboration], Phys. Rev. Lett. 102 (2009) 121801 [arXiv:0811.2512 [hep-ex]]. V. Büscher, talk at the EPS-HEP 2009 Conference, July 16-22, 2009, Cracow, Poland.

[23] M. Herbst, talk at the EPS-HEP 2009 Conference, July 16-22, 2009, Cracow, Poland.

[24] J. Kalinowski, R. Rückl, H. Spiesberger and P.M. Zerwas, DESY Internal Note [unpublished], 1997. S.Y.Choi et al. in progress.

[25] G. Aad et al. [The ATLAS Collaboration], 'Expected Performance of the ATLAS Experiment Detector, Trigger and Physics," arXiv:0901.0512 [hep-ex].

[26] G. L. Bayatian et al. [CMS Collaboration], "CMS technical design report, volume II: Physics performance," J. Phys. G 34 (2007) 995.

[27] K. Kawagoe and M. M. Nojiri, Phys. Rev. D 74 (2006) 115011 [arXiv:hep-ph/0606104].

[28] I. Hinchliffe et al., Phys. Rev. D 55 (1997) 5520.

[29] B. K. Gjelsten, D. J. Miller and P. Osland, JHEP 0412 (2004) 003.

[30] M. M. Nojiri, G. Polesello and D. R. Tovey, JHEP 0603 (2006) 063.

[31] K. Desch, J. Kalinowski, G. Moortgat-Pick, M. M. Nojiri and G. Polesello, JHEP 0402 (2004) 035. 
[32] J. L. Feng, K. T. Matchev and T. Moroi, Phys. Rev. D 61 (2000) 075005. J. L. Feng and F. Wilczek, Phys. Lett. B 631 (2005) 170.

[33] K. Desch, J. Kalinowski, G. Moortgat-Pick, K. Rolbiecki and W.J. Stirling, JHEP 0612 (2006) 007 [arXiv:hep-ph/0607104].

[34] J. A. Aguilar-Saavedra et al., Eur. Phys. J. C 46 (2006) 43. See also J. Kalinowski, Supersymmetry Parameter Analysis, Proc. Int. Conference on Supersymmetry and Unification of Findamental Interactions SUSY02 (Hamburg 2002), arXiv:hep-ph/0212388.

[35] B. C. Allanach, G. Belanger, F. Boudjema and A. Pukhov, JHEP 0412, 020 (2004).

[36] M. M. Nojiri, G. Polesello and D. R. Tovey, arXiv:hep-ph/0312317. K. Kawagoe, M. M. Nojiri and G. Polesello, Phys. Rev. D 71 (2005) 035008.

[37] H. C. Cheng, K. T. Matchev and M. Schmaltz, Phys. Rev. D 66 (2002) 056006.

[38] A. J. Barr, Phys. Lett. B 596 (2004) 205.

[39] J. M. Smillie and B. R. Webber, JHEP 0510 (2005) 069.

[40] C. Athanasiou, C. G. Lester, J. M. Smillie and B. R. Webber, JHEP 0608 (2006) 055. L. T. Wang and I. Yavin, JHEP 0704 (2007) 032 [arXiv:hep-ph/0605296].

[41] J.A. Aguilar-Saavedra et al., TESLA Technical Design Report, DESY 01-011 and arXiv:hep-ph/0106315; T. Abe et al. [American LC WG], in Proceedings of the APS/DPF/DPB Summer Study on the Future of Particle Physics (Snowmass 2001), SLAC-R-570 and arXiv:hep-ex/0106055-58; T. Abe et al. [Asian LC WG], KEK-Report-2001-011 and arXiv: hep-ph/0109166.

[42] G. Weiglein et al. [LHC/LC Study Group], arXiv:hep-ph/0410364.

[43] http://www.linearcollider.org

[44] http://www.desy.de/conferences/ecfa-lc-study.html

http://acfahep.kek.jp

http://physics.uoregon.edu/ lc/alcpg

[45] J. Kalinowski, arXiv:hep-ph/0508167. J. Kalinowski, arXiv:hep-ph/0309235.

[46] A. Freitas et al., arXiv:hep-ph/0211108. A. Freitas, Ph.D. thesis, Hamburg (2002), DESY THESIS-2002-023.

[47] H. U. Martyn, arXiv:hep-ph/0406123.

[48] H. U. Martyn, arXiv:hep-ph/0302024.

[49] G. A. Moortgat-Pick et al., Phys. Rept. 460 (2008) 131 [arXiv:hep-ph/0507011].

[50] M. Carena, A. Finch, A. Freitas, C. Milstene, H. Nowak and A. Sopczak, Phys. Rev. D 72 (2005) 115008.

[51] A. Freitas and A. von Manteuffel, arXiv:hep-ph/0211105.

[52] S. Y. Choi, J. Kalinowski, G. A. Moortgat-Pick and P. M. Zerwas, Eur. Phys. J. C 22 (2001) 563 [Addendum-ibid. C 23 (2002) 769].

[53] J.A. Aguilar-Saavedra, Phys. Lett. B596 (2004) 247.

[54] J.A. Aguilar-Saavedra and A.M. Teixeira, Nucl. Phys. B675 (2003) 70. J.A. Aguilar-Saavedra, LC-TH-2003-098 [hep-ph/0312140]. 
[55] S. Y. Choi, B. C. Chung, J. Kalinowski, Y. G. Kim and K. Rolbiecki, Eur. Phys. J. C 46 (2006) 511.

[56] S. Heinemeyer, S. Kraml, W. Porod and G. Weiglein, JHEP 0309 (2003) 075.

[57] For a review and references see e.g. V. I. Telnov, Acta Phys. Polon. B 37 (2006) 1049.

[58] I. Alvarez Illan and K. Monig, DESY LC note LC-PHSM-2005-002.

[59] P. Niezurawski, A. F. Zarnecki and M. Krawczyk, Acta Phys. Polon. B 37 (2006) 1187;

[60] P. Niezurawski, A. F. Zarnecki and M. Krawczyk, Acta Phys. Polon. B 36 (2005) 833. S. Y. Choi, J. Kalinowski, Y. Liao and P. M. Zerwas, Eur. Phys. J. C 40 (2005) 555, and arXiv:hep-ph/0508121.

[61] S. Y. Choi et al, Phys. Lett. B 606 (2005) 164.

[62] E. Accomando et al. [CLIC Physics Working Group], arXiv:hep-ph/0412251.

[63] M. M. Alsharoa et al. [Muon Collider/Neutrino Factory Collaboration], Phys. Rev. ST Accel. Beams 6 (2003) 081001.

[64] P. M. Zerwas et al., arXiv:hep-ph/0211076. C. G. Lester, M. A. Parker and M. J. White, JHEP 0601 (2006) 080 [arXiv:hep-ph/0508143].

[65] N. Arkani-Hamed, G. L. Kane, J. Thaler and L. T. Wang, JHEP 0608 (2006) 070.

[66] P. Skands et al., JHEP 0407 (2004) 036.

[67] M. Battaglia et al., Eur. Phys. J. C 33 (2004) 273.

[68] P. Bechtle, K. Desch and P. Wienemann, Comput. Phys. Commun. 174 (2006) 47. R. Lafaye, T. Plehn and D. Zerwas, arXiv:hep-ph/0404282.

[69] M. Battaglia, T. Barklow, M. Peskin, Y. Okada, S. Yamashita and P. Zerwas, arXiv:hep-ex/0603010.

[70] E. A. Baltz, M. Battaglia, M. E. Peskin and T. Wizansky, Phys. Rev. D 74, 103521 (2006) [arXiv:hep-ph/0602187]. 